\title{
Expert anticipation from deceptive action
}

\author{
Shuji Mori • Takuro Shimada
}

Published online: 22 February 2013

(C) Psychonomic Society, Inc. 2013

\begin{abstract}
Expertise in sports enhances the ability to anticipate forthcoming events from the observation of a player's actions. In the present study, we investigated whether this ability is applicable to deceptive action. In three experiments, performance at anticipating the direction change of a running opponent was examined with experienced rugby players and novice counterparts. These experiments were conducted with reaction-time and temporal-occlusion tasks, in combination with eye movement recordings and the presentation of filmed actions and their point-light representations. The main finding was that the experienced players were superior to the novices in their anticipation of deceptive actions, although their performance was still impaired by the deception, in comparison with their anticipation of nondeceptive actions. We also found that the experienced players anticipated nondeceptive actions less accurately than the novices, suggesting that the players' expectations of deceptive actions worked negatively on their judgments of nondeceptive actions. The results obtained with the pointlight representations closely resembled those obtained with the filmed sequences, indicating that anticipation was based on the kinematics of the running action. These results are discussed in the context of recent developments in research on expertise and deception in sports.
\end{abstract}

Keywords Anticipation - Expertise · Deception - Sports · Running

\section{S. Mori $(\triangle)$}

Department of Informatics, Faculty of Information Science and Electrical Engineering, Kyushu University,

744 Motooka, Nishi-ku,

Fukuoka City, Fukuoka 819-0395, Japan

e-mail: mori@inf.kyushu-u.ac.jp

T. Shimada

AP Innovation Division, Social Infrastructure Systems Unit,

Solutions Business Unit, Fujitsu Ltd., Tokyo, Japan
The ability to anticipate forthcoming events plays important roles in human perception and action. Anticipating objects at a specific time and/or location improves detection, discrimination, identification, and response to those objects (Coull \& Nobre, 1998; Nobre, Correa, \& Coull, 2007; Posner, 1980; Wright \& Ward, 2008). Anticipation of the occurrence of likely components operates in the perception of music (Janata \& Reisberg, 1988; Krumhansl \& Shepard, 1979), speech (Osterhout \& Holcomb, 1992), and bodily movements, such as lifting weights or delivering a tennis serve (Jones \& Miles, 1978; Runeson \& Frykholm, 1983).

Successful anticipation requires the detection and utilization of relevant information, or cues, about the target (Kunde, Elsner, \& Kiesel, 2007; Posner, 1980; Williams, Davids, \& Williams, 1999). These cues may be experimentally induced stimulation (e.g., an illuminated box or an arrow) or may be naturally contained in spatiotemporal sequences (e.g., music or bodily movement) that also contain the target. It is helpful, if not necessary, to know the essential structure of the spatiotemporal relations between the cue and target to allow for cue detection and utilization. Such knowledge is acquired through practice and experience (Kunde et al., 2007). Experts, who are well learned in a specific type of task and are considered to have extensive knowledge of the task, are superior in taskrelevant anticipation to nonexperts. Experts' superior anticipation is well documented in music perception (Besson \& Faiita, 1995; Loui \& Wessel, 2007) and the perception of sports actions (Williams et al., 1999).

Much of the aforementioned research has concerned anticipation from cues containing only veridical information. In everyday situations, we often encounter acts containing deceptive information that is intended to mislead us. Lie detection research has demonstrated that people are not very good at detecting lies (Bond \& DePaulo, 2006). The susceptibility to deception has also been observed in judgments of bodily action (Runeson \& Frykholm, 1983; Sebanz \& Shiffrar, 2009). 
In the present study, we investigated the interplay of anticipation and deception in sports expertise. Sports expertise is a particularly rich field of research on anticipation from bodily motion, and studies of situations involving deceptive action have recently accumulated (Brault, Bideau, Kulpa, \& Craig, 2012; Cañal-Bruland \& Schmidt, 2009; Dicks, Button, \& Davids, 2010a; Jackson, Warren, \& Abernethy, 2006; Rowe, Horswill, Kronvall-Parkinson, Poulter, \& McKenna, 2009). Here, we will first review the existing research on anticipation among sports players, including the methodologies typically used in the research and recent studies involving deception. Then we will present an overview of the present study.

\section{Anticipation in sports expertise}

It is now established that experienced sports players are superior to their less-experienced or novice counterparts in anticipating the outcomes of the observed actions of opponents (Williams et al., 1999). The faster and more accurate anticipation of experienced players has been experimentally confirmed in a variety of sports, including badminton (Abernethy \& Russell, 1987a, b), squash (Abernethy, 1990), tennis (Jones \& Miles, 1978), soccer (Williams \& Davids, 1998), and karate (Mori, Ohtani, \& Imanaka, 2002). Research now focuses on the spatiotemporal properties of advance cue utilization by experienced players - that is, where in the opponent's body and when in the sequence of the opponent's actions the players extract information relevant to their successful anticipation.

The temporal properties of cue utilization by sports players are usually examined with reaction-time (RT) and temporal-occlusion paradigms. RTs are measured when the observers are presented with dynamic presentations of play actions and required to decide, as quickly and accurately as possible, which of the possible outcomes will occur as a result of the action. The RT paradigm is favored in sports research because of its resemblance to real-life sports scenes: Players are often required to anticipate and decide what to do in a very limited time, and their characteristic ability in such situations will be manifested in the RT paradigm (Williams et al., 1999). Studies generally show that experienced players respond faster than their inexperienced counterparts, with no indication of a speed-accuracy trade-off (Helsen \& Starkes, 1999; Mori et al., 2002; Paull \& Glencross, 1997; Ward, Williams, \& Bennett, 2002; Williams \& Davids, 1998). The results are interpreted as indicating faster anticipation by experienced players. On the other hand, when expert players are allowed to respond with their skilled sports actions, they wait longer to respond than novices do, in order to minimize errors (Brault et al., 2012; Dessing \& Craig, 2010). Some studies have also reported that the players are no faster than their inexperienced counterparts in RTs to generic stimuli unrelated to the sport in question (e.g., a white dot), which supports the idea that the players' faster RTs to sports actions reflect superior anticipation specific to their expertise in the sport, not a speedup in their primitive sensory-motor functions (Mori et al., 2002; Williams \& Davids, 1998).

A major disadvantage of the RT paradigm for studying the temporal aspects of players' anticipation is that it does not allow for specification of the temporal course over which the experienced players extract anticipatory cues; we can only approximate the timing of cue extraction from the observed RTs, with some ungrounded assumptions about cognitive processing time (Mori et al., 2002). Alternatively, the temporal-occlusion paradigm is used to specify the temporal course of cue extraction. As in the RT paradigm, the observers are presented with dynamic presentations of play actions, but the presentations are terminated and replaced with blank displays at one of various temporal points, and the observers are required to predict the outcome of the action. The dependent variable is a measure of response accuracy, such as the proportion of correct responses, at each temporal point of occlusion. Studies typically show that experienced players anticipate above chance and more accurately than novices, prior to the point at which the action outcome is visible afterward-for instance, the ball-racket impact in a tennis serve (Farrow, Abernethy, \& Jackson, 2005; Jones \& Miles, 1978) or badminton stroke (Abernethy \& Russell, 1987a, b), or the ball-foot contact in soccer penalty kicks (Williams \& Burwitz, 1993). The player-novice difference diminishes after that point. Such results are interpreted as evidence that experienced players are able to extract anticipatory cues in advance (Williams et al., 1999) and that their superiority over novices in anticipation is more evident as the information available from the play scene becomes temporally more limited.

Spatial aspects of advance cue extraction have been studied with a spatial-occlusion paradigm and eye movement recordings. In the spatial occlusion paradigm, the observers are presented with play actions, as in the temporal-occlusion paradigm, but the presentations are partially masked. The observer is asked, after viewing the presentation, to predict the outcome of the action. A general assumption is that prediction accuracy is significantly lowered when the masked region contains anticipatory cues used by the observer. The spatial-occlusion paradigm has been used to identify characteristic locations of the cues utilized by expert players, in comparison with novice counterparts, for a variety of sports, including badminton (Abernethy \& Russell, 1987a, b), tennis (Shim, Carlton, \& Kwon, 2006), squash (Abernethy, 1990), soccer (Williams \& Davids, 1998), and fencing (Hagemann, Schorer, Cañal-Bruland, Lotz, \& Strauss, 2010). 
Eye movement recordings provide data regarding the spatial as well as temporal aspects of cue extraction by expert players. The observer's eye movements are recorded while he or she views and responds to dynamic presentations of play actions in a manner similar to when he or she encounters the actions in the field. The eye movement data are analyzed to provide the observer's fixation pattern, in terms of fixated locations and durations of viewing during the presentation (see Williams et al., 1999, for a review of measurement methodologies). It is hypothesized that the fixation pattern reflects the observer's visual-search strategies for information relevant to performing the task. Studies have reported different search strategies being employed by experienced players and less experienced counterparts, coupled with their performance differences in the concurrent perceptual-motor task (for reviews, see Cauraugh \& Janelle, 2002; Hagemann et al., 2010; Williams et al., 1999). For example, Williams and Davids (1998) identified situationdependent differences in visual search between experienced and inexperienced soccer players (see also Williams, Davids, Burwitz, \& Williams, 1994). They set up experimental conditions simulating one-on-one and three-on-three situations in soccer games and asked the participants to act as if they were defensive players attempting to intercept the pass of the offensive player, and to move their bodies accordingly. In the one-on-one situation, the experienced players fixated more frequently on the midbody region of the offensive player than did the inexperienced players. In the three-on-three situation, there was no difference between the two groups, who fixated most time on the lower body of the central, ball-possessing player. Williams and Davids also showed that these fixation patterns did not match with spatial-occlusion results, which were obtained from virtually the same participants and filmed sequences, except that the sequences were partially masked. In the one-on-one situation, masking either the hip or the legs and ball had detrimental effects on the anticipatory performance of both groups of participants, with no difference in effect size between the masked regions. In the three-on-three situation, masking areas other than the ball-possession player impaired the performance of the experienced players more than that of the inexperienced players. These results suggest that the experienced players extracted information in areas other than those on which they fixated most during the task. Other studies have also reported discrepancies between fixation and spatial-occlusion results (Abernethy \& Russell, 1987b; Hagemann et al., 2010). We may need to distinguish visual-search strategies, indicated by fixation data, from information extraction processes, inferred from spatial-occlusion data (Williams \& Davids, 1998). Visual-search strategies concern the optimal placement of the fovea to collect relevant information over the display, whereas attention can be oriented to areas outside of the fixation, and information will be extracted from those areas (Posner, 1980).

Spatial aspects of anticipation are also investigated with motion analysis of the players' actions that are the target of anticipation. Research has shown that despite large interand intraindividual differences in motion, some local and global movements of the player's body significantly relate to the outcome of the action (Brault, Bideau, Craig, \& Kulpa, 2010; Brault et al., 2012; Diaz, Fajen, \& Phillips, 2012; Huys, Smeeton, Hodges, Beek, \& Williams, 2008). The reliability of those movements increases as the action advances in time (Brault et al., 2012; Diaz et al., 2012), and correspondences between such reliable movements and the anticipation performance of experienced players observing the action suggest that players would be likely to fixate on those movements and extract anticipatory information from them (Huys et al., 2008). It has further been suggested that expert players act in parallel with the dynamics of their opponent's action (Brault et al., 2012). We will return to the latter point in the next section.

The studies so far reviewed have mostly used filmed sequences of play actions taken from a player's perspective on the field, with some exceptions in which virtual reality presentations of play scenes have been presented from the viewpoint of the moving observer, updated in real time (Brault et al., 2012; Dessing \& Craig, 2010). Recently, point-light presentations of a player's motion have gained popularity in sports anticipation research. As in walking and other biological actions (Johansson, 1973; for a review, see Blake \& Shiffrar, 2007), we perceive realistic movements of sports players' actions from the coherent motions of 10 to 20 points representing the major joints and body parts of the acting player. A major advantage of using point-light presentations in sports anticipation research is that they keep the kinematic properties of the acting player's motion largely intact, while excluding all contour and surface information of the body. This allows us to ascertain whether and to what extent expert superiority in anticipation relies on an opposing player's "motion" per se. Studies have shown that point-light presentations result in only a small reduction in anticipation accuracy as compared with filmed or virtualreality presentations (Abernethy, Gill, Parks, \& Packer, 2001; Shim et al., 2006; Vignais et al., 2010; Ward et al., 2002), and more importantly, the patterns of results obtained with these two types of presentation have been similar to each other in terms of expert-novice differences in the effects of spatial and temporal occlusions (Abernethy \& Zawi, 2007; Abernethy, Zawi, \& Jackson, 2008; Shim et al., 2006) and in visual-search strategies (Ward et al., 2002). This offers strong evidence that the information extracted by experienced players as anticipatory cues is largely kinematic.

In sum, converging evidence has shown that expert sports players extract anticipatory cues in advance from specific 
sources, including the kinematic motions of the opposing player's body components. Our review of past research has also shown advantages and disadvantages in the experimental paradigms used and discrepancies in the findings obtained with them. One therefore needs to use more than one paradigm, or to use paradigms other than those that have been used in past research, in order to take all of the findings into consideration and provide an overview of expertise anticipation.

\section{Anticipation from deception}

Extending the accumulated knowledge of anticipation in sports expertise, Jackson et al. (2006) investigated the effects of a sidestep on anticipation among rugby players attempting to tackle opposing players running toward them with balls. To avoid being tackled, a runner will sidestep to his left or right before running round the tackling players in the opposite direction. Jackson et al. videotaped rugby players running toward a camera and taking either a sidestep or no step, and they presented the films to expert rugby players and novices using the temporal-occlusion task. The results showed that the two groups of participants were equally accurate in judging the no-sidestep stimuli, while the judgment accuracy of the sidestep stimuli was lower only for the novices, not for the players. The players judged the two types of stimuli indifferently. These results suggest that the expert rugby players were less susceptible to deceptive motions than were the novices.

Subsequent studies have confirmed the superior performance of expert players over novices in situations involving deception (Cañal-Bruland \& Schmidt, 2009; Dicks et al., 2010a; Rowe et al., 2009; Sebanz \& Shiffrar, 2009). In contrast to Jackson et al. (2006), however, some of the studies found that players were also susceptible to deception. Rowe et al. showed that expert tennis players were less accurate in anticipating ball directions from deceptive groundstrokes than from genuine ones, although the players performed above chance and better than novices for both types of groundstroke. Dicks et al. (2010a), who constrained the viewing time of expert soccer goalkeepers attempting to save penalty kicks on the field, found that the goalkeepers' saving performance was impaired when they were unable to watch the penalty kicker's approach motion toward the ball, to a larger degree for kicks with deceptive motion than without deception.

Recently, Brault and his colleagues conducted a series of studies combining biomechanical analysis and anticipation tasks related to rugby players' sidesteps (Brault et al., 2010, 2012). In their biomechanical analysis, Brault et al. (2010) analyzed the running motion of expert rugby players who changed their direction, with or without a sidestep, in an attempt to escape from being tackled by a real defender (cf. Jackson et al., 2006) who tried to intercept the running player. The analysis revealed the distinguishing features of deceptive running motion involving a sidestep, as compared with the nondeceptive motion: The angular change of the head and upper trunk started earlier and became larger for the deceptive than for the nondeceptive motion. The placement of the nonstepping foot showed a similar pattern. In contrast, the angle of the lower trunk and the center of mass, which represented the global motion direction of the whole body, started to change earlier and to a lesser degree for the nondeceptive motion than for the deceptive motion. These results imply that the deceptive running motion of rugby players is a combination of minimizing honest signals - that is, the center of mass and lower trunk movements, which are mechanically related to the genuine running direction-and exaggerating deceptive signals, such as the head, the upper body, and the nonstepping foot (Brault et al., 2012).

In their anticipation tasks, Brault et al. (2012) set up an immersive and interactive virtual reality environment in which the participants, expert rugby players and their novice counterparts, were presented with a 3-D image of a virtual rugby player for which running actions had been created from the biomechanical analysis of a real player's motion (Brault et al., 2010). In one experiment, the temporalocclusion paradigm was used with the virtual player's running actions being occluded at $0,100,200$, or $300 \mathrm{~ms}$ after the first footstep for changing direction. The results showed that the expert players judged the running direction almost perfectly for the no-sidestep action but were significantly less accurate for the sidestep action: Their accuracy was lowest at the 0 -ms occlusion point and increased as the occlusion point advanced, up to a level comparable to that for the no-sidestep action at the $300-\mathrm{ms}$ point. The novices showed a similar pattern, but their accuracy for the sidestep action was lower than that of the players, particularly at early occlusion points. This suggests that the expert rugby players and the novices were both susceptible to deception (cf. Jackson et al., 2006).

To see whether and how their anticipation from the sidestep action would be related to the mechanical motion of the action, Brault et al. (2012) conducted a regression analysis and fitted logistic functions of the accuracy data at each occlusion point to the corresponding biomechanical parameters tau, which represent unfolding patterns of body parts of the running player relative to the point of direction change. Among the various body parts, the center of mass yielded the best fit for the rugby players, and the upper trunk angle for the novices. This suggests that the expert rugby players were tuned into the honest signals (center of mass), whereas the novices were tuned into the deceptive signals (upper trunk). The best-fitting functions for the center of mass were used to estimate the time at which judgment 
accuracy reached $50 \%$ on the basis of center-of-mass information, and the estimated times for the expert rugby players and the novices were 183 and $18 \mathrm{~ms}$, respectively, before the moment of direction change. This indicates that the honest signals led the expert rugby players to anticipate better than the novices, well before the direction change, which conformed to the expert-novice differences in the occlusion data.

In another experiment, Brault et al. (2012) had their participants move as if to intercept the virtual player, and their center-of-mass motion was analyzed. For the expert rugby players, the tau of center of mass was more highly correlated with the tau of the virtual player's center of mass than of the virtual player's upper trunk, whereas the pattern was reversed for the novices. This corroborated the occlusion data, in that the expert rugby players were tuned into the honest signals and the novices into the deceptive signals. Brault et al. (2012) also found that the expert players were significantly slower than the novices in initiating the interceptive motion, but were more accurate in making the motion in a correct direction toward the escaping player. Brault et al. (2012) interpreted this finding as evidence for the expert rugby players' superiority over the novices in minimizing the number of motion errors in a wrong direction.

In sum, other than Jackson et al. (2006), most studies have found that expert players are susceptible to deception. Particularly suggestive are the studies of Brault et al. (2010, 2012), who demonstrated a close relation between the mechanics of deceptive motion and the spatiotemporal features of expertise anticipation from the deception.

\section{Overview of the present study}

In the present study, we further investigated the spatiotemporal features of rugby players' anticipation of direction change in opponents' running, with or without sidesteps. For this purpose, we conducted three experiments, using the RT paradigm with eye movement recordings (Exp. 1), the temporal-occlusion paradigm (Exp. 2), and temporal occlusion using point-light displays (Exp. 3).

In Experiment 1, we employed the RT paradigm (Exp. 1) to investigate the temporal features of anticipation. We were particularly interested in examining two predictions drawn from the results of Brault et al. $(2010,2012)$. One prediction, derived from their finding of estimated time from the best-fitting functions for the center of mass, was that expert rugby players would be able to make above-chance anticipation much faster than novice counterparts. The other prediction was based on the initiation times of intercepting actions, which were slower but more accurate for the expert players than for the novices: According to this prediction, expert rugby players would wait longer to respond, in order to avoid premature errors. Note that the differences between these two predictions lies not only in the speed but also the accuracy of anticipating a change of direction. In our experiment, we measured RTs with an emphasis on speed as well as accuracy when judging the change of direction, to see which of the two predictions the RT data would follow.

We also measured the participants' eye movements during the RT task of Experiment 1, to see whether their fixation patterns would corroborate Brault et al.'s (2010, 2012) hypothesis of the differential tunings of expert rugby players and novices to honest and deceptive signals. Assuming that rugby players and novices focus on their tuned signals in the opponent's body to extract anticipatory information regarding the opponent's change of direction, we predicted that the players would fixate most on the lower trunk of the opponent's body, whereas the novices would fixate more on the head, upper body, and nonstepping foot than on the other regions.

Experiment 2 was conducted with the temporal-occlusion paradigm, similar to the experiments of Jackson et al. (2006) and Brault et al. (2012), to enable a direct comparison of our results with theirs. We also employed the temporalocclusion task in Experiment 3, but this time using pointlight displays of running actions created from the videotaped stimuli used in the other experiments. The results could be used to assess the skills of our participants - that is, expert rugby players and novice counterparts-in perceiving the biological motions of running.

In all of the experiments of the present study, we included double-sidestep stimuli, in which a model player took two steps before changing direction (e.g., taking a step first to the left and next to the right before finally running to the left). In rugby games, double sidesteps are often used with one sidestep and no step. The mixed presentations of those three types of running actions should make the experimental setup more realistic and provide new insight into the expert rugby players' anticipations and reactions to the opponents' deception.

\section{Experiment 1}

In this experiment, we examined the spatiotemporal features of experienced rugby players' anticipations of an opponent's change of direction, made with or without sidesteps. In terms of temporal features, we were interested in whether the RT data would conform to two predictions drawn from the study of Brault et al. (2012) - that is, whether the rugby players would respond to the direction change relatively faster or slower than their novice counterparts. In terms of spatial features, we analyzed the participants' eye movements during the RT task to see whether their fixation pattern would corroborate Brault et al.'s $(2010,2012)$ hypothesis of differential tunings to honest and deceptive signals in the opponents' running actions. 


\section{Method}

Participants Ten collegiate rugby players and ten nonplayers or novices (mean ages, $23.2 \pm 1.1$ years and $22.3 \pm$ 0.5 years, respectively), all males, participated. The players had played rugby for a mean of $9.0 \pm 2.0$ years, with five of them having competed at national level in Japan. The novices were university students who had not played rugby at a competitive level, but all of whom had seen rugby played and possessed basic knowledge of the game. ${ }^{1}$ All participants had normal or corrected-to-normal visual acuity and color vision, and they gave written informed consent prior to the experiment.

Apparatus and stimuli A personal computer (Dell Precision 380) and color graphic system (Cambridge Research System VSG2/5) were used to control the experiment and generate stimuli that were projected onto a white screen $(150 \times$ $200 \mathrm{~cm}$ ) using a projector with a spatial resolution of 768 $\times 1,024$ pixels (Panasonic TH-LB60NT). A custom-made response box was used for participant responses.

The stimuli were video clips simulating the participant's view of a rugby player running toward the camera and suddenly changing direction to the left or right, with or without a sidestep (see Fig. 1). For the stimuli, we had running actions from three rugby players who had either competed at the national level or been selected to a regional top team in Japan, recorded by a digital video camera (Sony DCR-SR60) with a $30-\mathrm{Hz}$ sampling rate. The player started to run $16 \mathrm{~m}$ away from the camera, which was positioned at a height of $120 \mathrm{~cm}$, simulating the eye level of a defensive player making a tackle, and then changed direction either 4 or $5 \mathrm{~m}$ from the camera, ${ }^{2}$ simulating running around a

\footnotetext{
${ }^{1}$ Some of the novices in this and the subsequent two experiments of the present study had practiced rugby and played games in their highschool physical education class, so that they were more experienced with rugby than the other novices. We looked into their individual data and found that their performance did not differ significantly from the other novices or cause any significant interaction with the independent variables considered in any of the experiments reported here.

${ }^{2}$ The two different locations ( 4 and $5 \mathrm{~m}$ ) for directional change were used in the recording of running actions to prevent the observers from judging the actions on the basis of the relative timing (and/or the relative retinal size of the player at that timing) at which the running player made a sidestep. As can be seen in Fig. 1, the players performing actions with one and double sidesteps made their first sidesteps earlier than the player making no sidestep made his step for directional change. Half of the stimuli used in the experiment were recorded with directional changes at $4 \mathrm{~m}$, and the other half at $5 \mathrm{~m}$, and they were randomly presented across trials. Although these manipulations did not completely negate the relative timing of directional change as a cue indicating the type of action, in practice they were sufficient to make the timing unreliable for the observer's judgment. In retrospective interviews after the experiment, no participant reported having used the relative timing or retinal image size of the running player's directional change for his judgments.
}

virtual defensive player to avoid a tackle. The locations of the direction changes were indicated by marks on the ground that were not seen from the video camera. The players' running actions included no sidestep, one sidestep, or a double sidestep, the latter two movements being performed before the player changed direction. Figure 1 illustrates the three types of actions. For the no-sidestep action (left column), the player ran straight toward the camera and changed direction to the right. For the one-sidestep action (center column), the player made a sidestep to the right (at the frames from -200 to $-100 \mathrm{~ms}$ ) before changing direction to the left. For the double-sidestep action (right column), the player made a first sidestep to the right (from -400 to $300 \mathrm{~ms}$ ), a second sidestep to the left ( -200 to $-100 \mathrm{~ms}$ ), and finally changed direction to the right. The timing and location of sidesteps varied from action to action; we only specified the location of the final directional change. From the recordings of each of the three players, 20 actions were chosen as the stimuli to be presented in the following experiments: For each of two direction changes to the left and to the right, four actions included no sidestep, four included one sidestep, and two included a double sidestep. The selection of those actions was made by the second author of the present article, an experienced rugby player, on the basis of whether the moment of the footstep for direction change was clearly recorded within one video frame, and for the single- and double-sidestep actions, whether the sidestep appeared to be effective in obscuring the genuine running direction. A total of 60 stimuli were thus presented. Each stimulus was digitized and edited in the form of successive frames and presented at $30 \mathrm{~Hz}$ on the screen. The stimulus size projected on the screen was adjusted to match the retinal size of the running player viewed on the field.

The participants' eye positions were monitored by an infrared eye movement recording system (NAC model EMR-8B). The system had a temporal resolution of $16.67 \mathrm{~ms}$ (sampling rate $60 \mathrm{~Hz}$ ) and was able to measure eye movements linearly within the monitor of the system (an operating range of $32^{\circ} \times 24^{\circ}$ in the horizontal and vertical dimensions, respectively), with a spatial resolution of $0.1^{\circ}$ of visual angle. The images being viewed by the participants were simultaneously recorded by a charge-coupled-device (CCD) camera attached to the system, with a $30-\mathrm{Hz}$ sampling rate and a spatial resolution of $512 \times 492$ pixels over $44^{\circ} \times 33^{\circ}$ of visual angle.

Procedure The experiment was conducted in a dark room in which the participant was seated while viewing a screen binocularly from a distance of approximately $120 \mathrm{~cm}$. The projected stimuli subtended $65^{\circ} \times 80^{\circ}$ in height and width. An experimental session started with dark adaptation for 5 min, at which time the participants were given the following instructions: Decide, as quickly and accurately as 
Fig. 1 Examples of stimuli used in Experiments 1 and 2. Different expert players were shown performing running actions with no, one, and double sidesteps, respectively, with the images depicting every third frame from $500 \mathrm{~ms}$ before to $100 \mathrm{~ms}$ after the foot that initiated the final directional change contacted the ground $(0 \mathrm{~ms})$. Times in parentheses next to the rightmost column indicate the occlusion points for actions with double sidesteps in Experiment 2. See the text for details

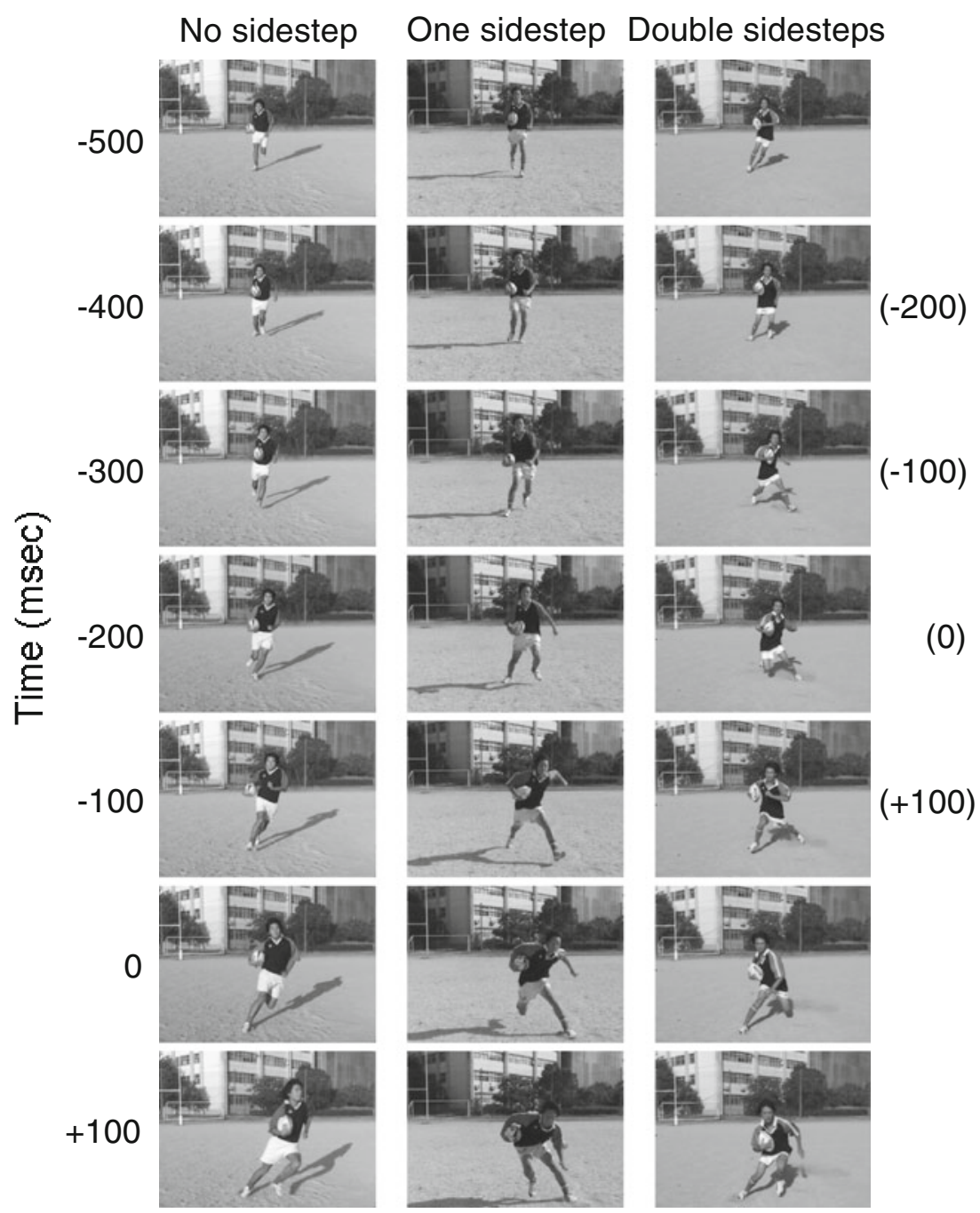

possible, whether the player shown on the screen was going to change direction to the left or right, pressing one button on the response box with the left hand for a direction change to the left, or the other button with the right hand for a change to the right; keep the proportion of correct responses above $80 \%$ while responding quickly, if possible before the completion of running action in the presented stimulus; the running actions would include, with a certain probability, sidesteps to deceive the viewer with regard to a directional change. On each trial, a white fixation point $1.3^{\circ}$ in diameter was presented first for $5,000 \mathrm{~ms}$ on a black background, followed by the stimulus presentation, which was terminated when the participant made a response. RT was measured as the time from which the runner's foot last touched the ground before making a directional change (corresponding to the frames at $0 \mathrm{~ms}$ in Fig. 1) to that at which the participant pressed the button. The next trial started immediately.

On each trial, horizontal and vertical eye positions during the stimulus presentation were recorded from the right eye of the participant. Before the experimental session, we asked the participants to fixate nine stationary points on the recording system monitor in order to calibrate the recording system. All of the eye position data were stored on a hard disk and analyzed offline.

Each participant completed an experimental session of six practice trials and 60 main trials. In the practice trials, the stimuli consisted of random presentations of directional changes to the left or the right with no sidestep, one sidestep, or a double sidestep. In the main trials, the stimuli consisted of random presentations of 60 different actions (see the Apparatus and Stimuli section) - that is, 24 with no sidestep, 24 with one sidestep, and 12 with a double sidestep. One half of the stimuli included a directional change to the left, and the other half were to the right.

Data analysis RTs faster than $100 \mathrm{~ms}$ were regarded as premature (Seya \& Mori, 2007, 2012), and trials with those RTs were removed from the subsequent analyses of RTs and proportions of correct responses. This resulted in the removal of $2.5 \%, 0.3 \%$, and $3.8 \%$ of trials for the no-sidestep, onesidestep, and double-sidestep actions, respectively. No RTs were longer than $800 \mathrm{~ms}$. A 2 (group: player or novice) $\times 3$ 
(action: no sidestep, one sidestep, or double sidestep) $\times 2$ (direction change: left or right) $\mathrm{ANOVA}^{3}$ was conducted separately on the individual data for RTs and proportions of correct responses. All post-hoc comparisons of the group differences and multiple comparisons with paired $t$ tests for repeated measures were conducted with the overall $\alpha$ adjusted to .05 by Bonferroni correction.

In the analysis of the eye movement data, we computed the percentage viewing time (Savelsbergh, Williams, van der Kamp, \& Ward, 2002; Ward et al., 2002), which is the percentage of time the participants spent fixating various areas of the stimulus display when performing the RT task (from the onset of stimulus presentation to the buttonpress). ${ }^{4}$ The areas considered in the analysis were the running player's head, chest, abdomen, hip, and legs (which were the regions from around midthigh to toe on both legs). Fixation data that did not fall into one of these areas were pooled into an "unclassified" category. The analysis was conducted on individual data by superimposing scanpaths over the images of the stimulus display that were recorded by a CCD camera. The data of two novice participants were found to be aberrant, due to equipment problems, and were discarded. The remaining individual data were subjected to a four-way ANOVA with Group as a between-participants factor and Action, Direction Change, and Fixation Location (head, chest, abdomen, hip, legs, and unclassified) as within-participants factors. Similarly for the RTs and the proportions of correct responses, all of the post-hoc comparisons and multiple paired $t$ tests were conducted with Bonferroni-corrected overall $\alpha$ levels of .05 .

\section{Results}

Figure 2A shows the mean correct RTs of the rugby players and the novices separately for the three types of actions and the two changes in direction. The players responded faster than the novices for all types of actions: When pooled across the two change directions, the mean RTs and standard errors (in parentheses) of the players and the novices were,

\footnotetext{
${ }^{3}$ In this and the subsequent ANOVAs reported in this article, the significance levels were computed using Huynh-Feldt corrected degrees of freedom for lack of sphericity in repeated measures.

${ }^{4}$ Other components of eye movements, such as search rate and search order, have also been reported in research on expertise perception in sports (Savelsbergh et al., 2002; Ward et al., 2002; for a review, see Williams et al., 1999). We were unable to extract those components from our eye position data because the image size of the running players in our stimuli expanded three- to fourfold during the presentation durations of 1,200-2,000 ms. An analysis of search rates and orders would require the extraction of fixations, which are defined as the eye remaining stationary within $1.5^{\circ}$ for $120 \mathrm{~ms}$ or longer (Savelsbergh et al., 2002). In our data, such "fixation" locations on the running player's body varied during the fixation, because the images of the player changed rapidly, which precluded extracting stable and meaningful patterns of visual search.
}
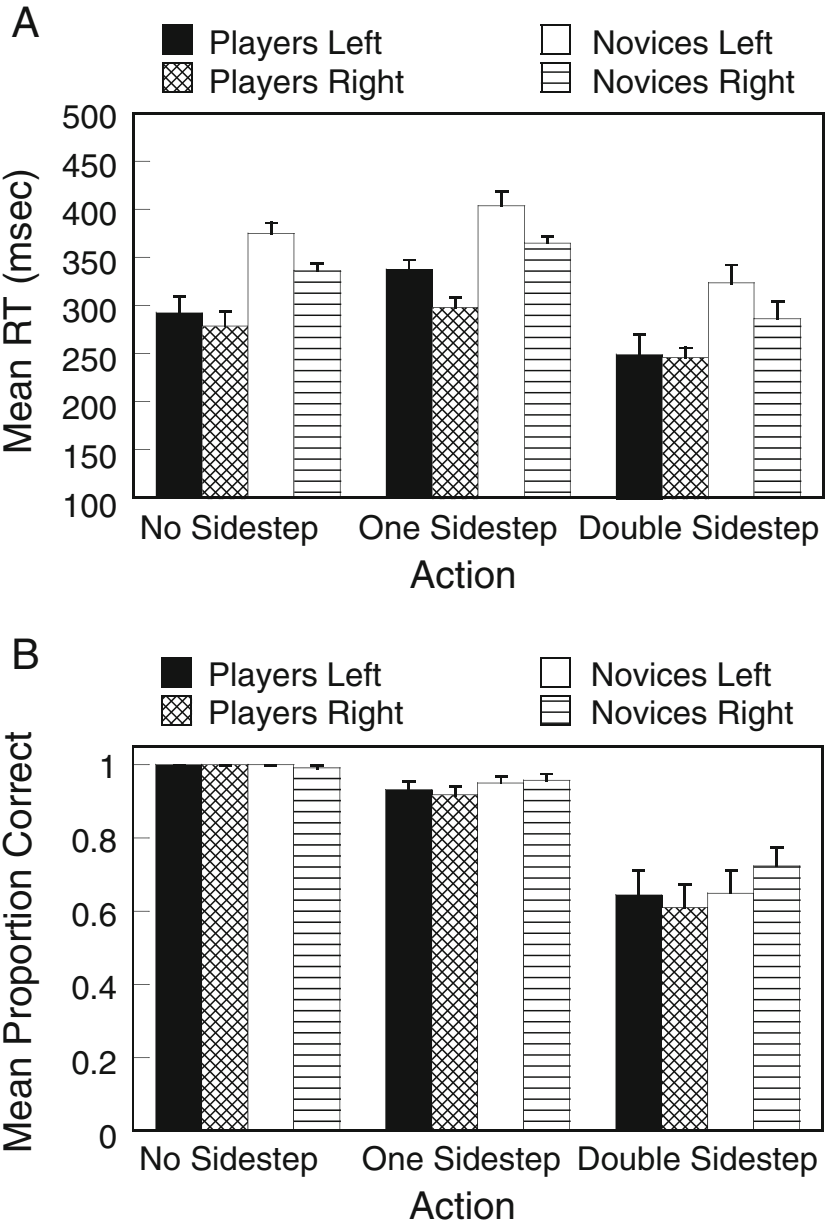

Fig. 2 Results of Experiment 1: (A) Mean RTs and (B) mean proportions of correct responses for ten rugby players and ten novices for different types of actions and change directions. The vertical bars indicate standard errors of the means

respectively, 286 (15.6) and 355 (9.9) $\mathrm{ms}$ for the nosidestep, 318 (10.0) and $384(9.9) \mathrm{ms}$ for the one-sidestep, and 247 (14.1) and 303 (17.8) $\mathrm{ms}$ for the double-sidestep actions. Both groups responded fastest to the double-sidestep action, and they responded slightly faster to the no-sidestep action than to the one-sidestep action. Note also that the RTs for direction change were faster for changes to the right than to the left, for both groups and all three types of actions. A threeway ANOVA showed that all of the main effects were significant [group, $F(1,18)=15.17, \eta_{\mathrm{p}}^{2}=.457$; action, $F(2,36)=$ $48.96, \eta_{\mathrm{p}}^{2}=.731$; direction, $F(1,18)=26.19, \eta_{\mathrm{p}}^{2}=.593$; all $p \mathrm{~s}<.01]$, but none of the interactions was significant. For the main effect of action, multiple comparisons showed significant differences between any two of the three types of actions.

Figure 2B shows the mean proportions of correct responses. The proportions were distinctively lower for the double-sidestep action than for the other two types of actions, which yielded quite high proportions. We found no noticeable difference due to the participant group or the 
direction change. A three-way ANOVA on the individual proportions of correct responses showed that only the main effect of action was significant, $F(2,36)=104.85, p<.01, \eta_{\mathrm{p}}^{2}=.853$. Multiple comparisons for action showed significant differences between any two of the three types of actions.

Figure 3 shows the mean percentage viewing times of the remaining eight novices and the ten players, separately for the

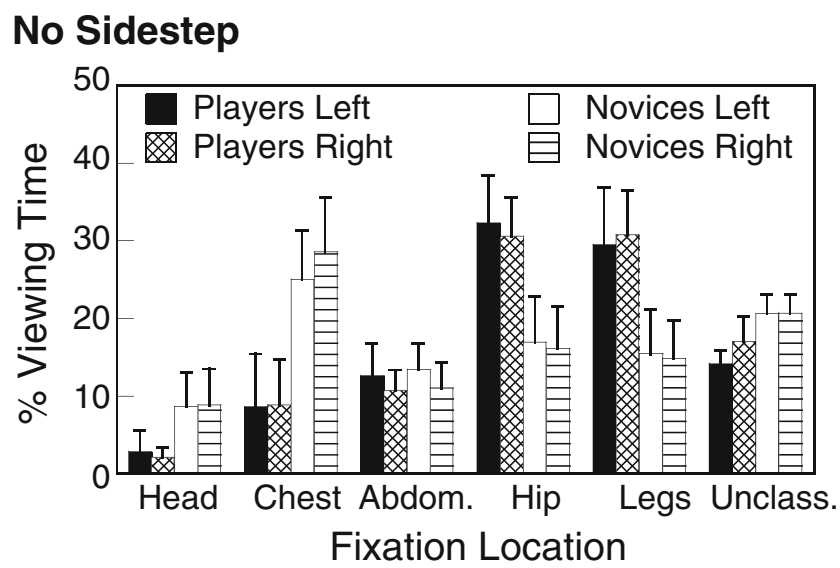

\section{One Sidestep}
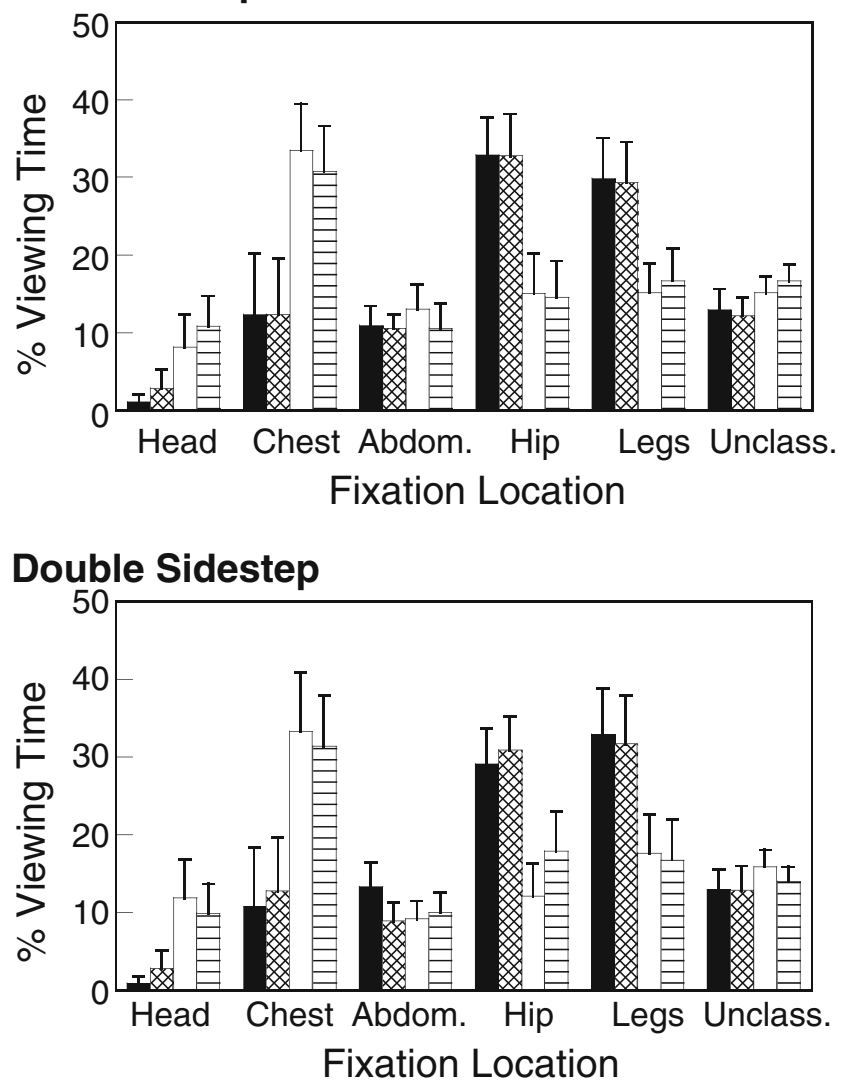

Fig. 3 Results of Experiment 1: Mean percentage viewing times per fixation location for ten players and eight novices, presented separately for the three types of actions. "Abdom." and "Unclass." refer to the abdomen and unclassified categories, respectively. The vertical bars indicate standard errors of the means three types of actions. Most notable was the difference in the high-percentage locations between the two groups. For the players, the percentages peaked at the hip and the legs, while the highest percentage for the novices was at the chest. For both groups, the percentage was lowest at the head. No apparent difference emerged between left and right direction changes. A four-way ANOVA showed a significant main effect of fixation location, $F(5,80)=4.18, p<.05, \eta_{\mathrm{p}}^{2}=.20$ 7 , with significant Group $\times$ Fixation, $F(5,80)=3.75, p<.05$, $\eta_{\mathrm{p}}^{2}=.190$, and Action $\times$ Fixation, $F(10,160)=3.89, p<.01$, $\eta_{\mathrm{p}}^{2}=.196$, interactions. No other main effect or interaction was significant. Post-hoc comparisons showed that the percentage at the hip was significantly higher for the players than for the novices, while the percentage at the chest was significantly higher for the novices. Multiple comparisons showed that for the players, the peak percentages at the hip and the legs were significantly different from the percentages at the head and the unclassified category. For the novices, the percentage at the chest was only significantly different from the percentage at the head. It seems that other differences among fixation locations were masked by variability due to the type of action. The percentage at the chest was significantly higher for the one-sidestep and double-sidestep actions than for the no-sidestep action, while the percentage at the unclassified category was significantly higher for the no-sidestep action than for the other two types of actions. No other differences among the three types of actions were significant at each fixation location.

\section{Discussion}

This experiment clearly showed faster judgments of the rugby players as compared to the novices concerning the directional change of opponents running toward them. The players responded faster than the novices for all types of actions, with no significant interaction with action type or speed-accuracy trade-off regarding the player-novice differences. This result conformed to the finding of Brault et al. (2012) that the estimated time of reaching above-chance anticipation was faster for rugby players than for novices.

We conducted a follow-up experiment, to see whether such faster responses of rugby players would take place in RT tasks in general - that is, whether rugby players would respond faster than novices, no matter what the stimuli and tasks were, due to their training for quick reactions. We had new groups of rugby players and novices, ${ }^{5}$ comparable to those who participated in this experiment, perform simple and choice RT tasks to a small black circle presented on the

\footnotetext{
${ }^{5}$ They were the same as those who participated in Experiment 2. They first performed in the simple and choice RT experiments and then, after taking a good rest, Experiment 2. See the Participants section of Experiment 2 for their personal information.
} 
white background of the CRT display. For either task, the mean RT of the player group was virtually identical to that of the novice group, with no significant difference between them. This followed the general consensus that sports players are no better than novice counterparts in their primitive sensory-motor functions, including simple and choice RTs (see the introduction), and confirmed that the rugby players' faster RTs in Experiment 1 were due to their advantage in anticipating the direction change of the running opponent.

RTs were fastest for the double-sidestep actions, second for the no-sidestep actions, and slowest for the one-sidestep actions. The double-sidestep actions may have been easy to respond to because the participants knew that there would be no further step and that they could determine the running direction as soon as the players in the stimuli made the third step. The one-sidestep actions were difficult because they contained movements very similar to those in the doublesidestep actions. The no-sidestep actions may have been distinguished from the other two types of stimuli because they were real rather than feigned. However, the fastest RTs for the double-sidestep actions may have been due to a speed-accuracy trade-off; the mean proportion of correct responses for the double-sidestep actions was below $80 \%$, despite the instructions given to the participants (see the Procedure section), and much lower than those for the other two types of action. In Experiment 2, we attempted to clarify the performance difference due to the three types of actions, using the temporal-occlusion method.

This experiment also showed, unexpectedly, that the RTs were faster for direction changes to the right than to the left. We carefully checked the apparatus and the stimuli used in this experiment, but found no anomaly that may have caused this difference. One possibility is that, since all of the player and novice participants were right handed, they responded to the direction change to the right with their dominant hand (see the Procedure section), which might have given an RT advantage for the right direction over the left direction. We checked this possibility in the aforementioned follow-up experiment, by computing the mean choice RTs of the right-handed participants (nine players and ten novices) separately for right-hand responses to the right-side presentations of the dot and for left-hand responses to the left-side presentations. For both the player and the novice groups, the mean RTs with the left and right hands were virtually identical and not significantly different from each other. This made it unlikely that the participants' right-handedness caused the RT advantage for the right direction changes in Experiment 1. We do not pursue this matter further, because we have no available answer at present, and this is not the central issue of the present study.

The fixation data were generally consistent with Brault et al.'s (2012) observation of differential tunings of rugby players and novices to honest and deceptive signals of an opponent's running action. One of the most fixated areas for the players was the opponent's hips, or lower trunk (honest signal), while the novices fixated most on the chest, or upper trunk (deceptive signal). We should also note that some discrepancies existed between the fixation patterns and Brault et al.'s (2012) tuning hypothesis. In Experiment 1, the other peak of the fixation data for the players was at the opponent's legs (from midthigh to toe in both legs), which was not regarded as an honest signal. The novices, as well as the players, spent the least time fixating on the head, which was identified as one of the deceptive signals in Brault and colleagues' (Brault et al., 2010, 2012) studies. We have no ready answer for these discrepancies, but one possibility is due to the technical difficulty of analyzing fixation locations in the present experiment. Since the image size of the running players in our stimuli expanded three- to fourfold during presentation durations of 1,200-2,000 ms, fixation locations on the running player's body varied during the fixations due to the rapid expansion of the image size of the body. This might have introduced variability, and possibly errors, in our identification of the fixation locations.

\section{Experiment 2}

In Experiment 2, we used a temporal-occlusion task to investigate the temporal aspects of experienced rugby players' anticipations of an opponent's running direction. Experiment 1 provided data concerning the player-novice differences in making quick decisions about the opponent's action with and without sidesteps, although the data were partially confounded with a speed-accuracy trade-off. The temporal occlusion task would allow for specification of the temporal course of the player-novice differences in anticipation (see the introduction) and also provide data that could be used for direct comparison with the findings of Jackson et al. (2006) and Brault et al. (2012).

\section{Method}

Ten collegiate rugby players and ten novices (mean ages, $21.4 \pm 0.8$ years and $22.3 \pm 0.6$ years, respectively), all males, participated. They were all different from those who participated in Experiment 1. The players had played rugby for a mean of $9.2 \pm 2.1$ years, with five of them having competed at the national level or been selected for a regional top team in Japan. The novices were university students who had not played rugby at a competitive level, but all of whom had seen rugby played and possessed basic knowledge of the game. All of the participants had normal or corrected-to-normal acuity and color vision, and they gave written informed consent prior to the experiment. 
They participated in the simple and choice RT experiment (see note 5) before participating in this experiment.

The apparatus was the same as for Experiment 1, except that a different personal computer (Compaq nx6320) was used. The stimuli were identical to those of Experiment 1, except that their presentation was occluded at one of five temporal points. Figure 1 indicates those occlusion points to the left and the right of the columns. For the no-sidestep and one-sidestep stimuli, the occlusion points were determined relative to the last time that the runner's foot touched the ground to make a directional change, and they were -300 , $200,-100,0$ (the moment that the foot touched the ground), and $100 \mathrm{~ms}$. For the double-sidestep stimuli, the $0-\mathrm{ms}$ point was the moment of the second footstep (one step before making the final directional change) touching the ground, and the five occlusion points were $-200,-100,0,100$, and $200 \mathrm{~ms}$. Therefore, the occlusion points for both the onesidestep and the double-sidestep stimuli were set relative to the moment of the runner making the second step, and we were able to analyze the participants' judgments for those two types of stimuli on the same temporal scale.

The procedure was similar to that of Experiment 1, with the following changes. The participants were instructed to predict the directional change (left or right) after the stimulus was occluded. In each trial, the stimulus was presented up to the time of one of the five occlusion points, after which the stimulus disappeared and a response window was presented. The participant used a computer mouse to indicate "left" or "right" on the window corresponding to his answer. The participants were given no time constraint for responding, but were told not to take too much time making their decision. Each participant completed an experimental session of three to six practice trials and 300 main trials, in which the stimuli consisted of random presentations of the combinations of the 60 different actions used in Experiment 1 and five occlusion points. Brief rest periods were given whenever requested.

\section{Results}

Figure 4 shows the mean proportions of correct responses of the rugby players and the novices as a function of occlusion point, separately for the three types of stimuli. Performance was markedly different among the three types of stimuli. For the no-sidestep stimuli, the proportions correct were above chance level (.5) and gradually increased as the occlusion point advanced. Interestingly, the novices were more accurate, although only slightly, than the players up to the 0 -ms occlusion point, after which the performances of both groups were almost perfect (1.0). For the one-sidestep
Fig. 4 Results of Experiment 2: Mean proportions of correct responses of ten rugby players and ten novices, presented separately for the three types of actions. The vertical bars indicate standard errors of the means. For the double-sidestep actions (bottom panel), the mean proportions of errors for one sidestep are also shown as thin continuous and dotted lines, corresponding to the performance of the players and the novices, respectively
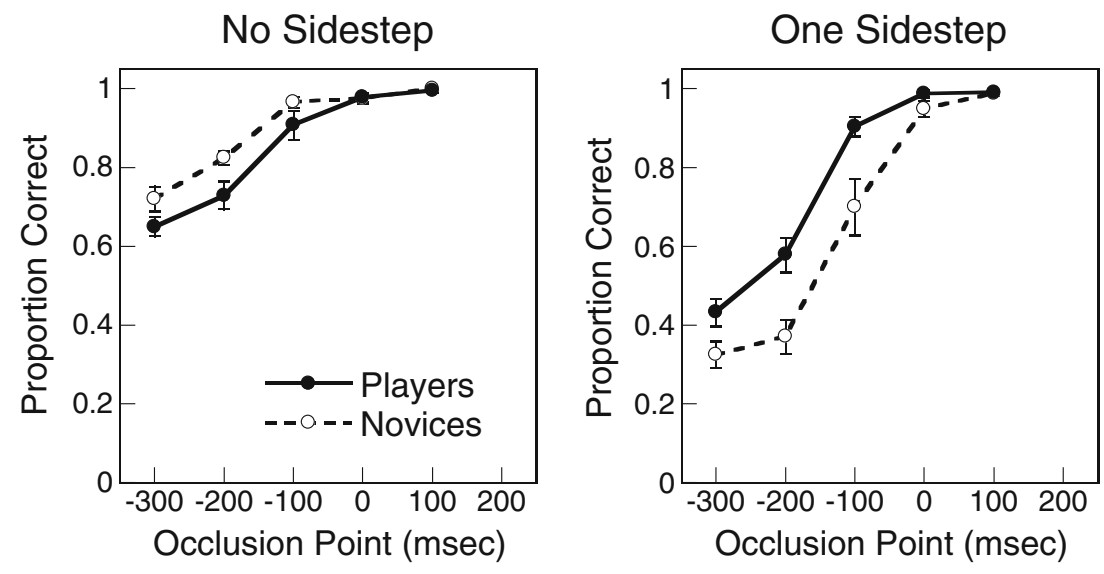

\section{Double Sidestep}

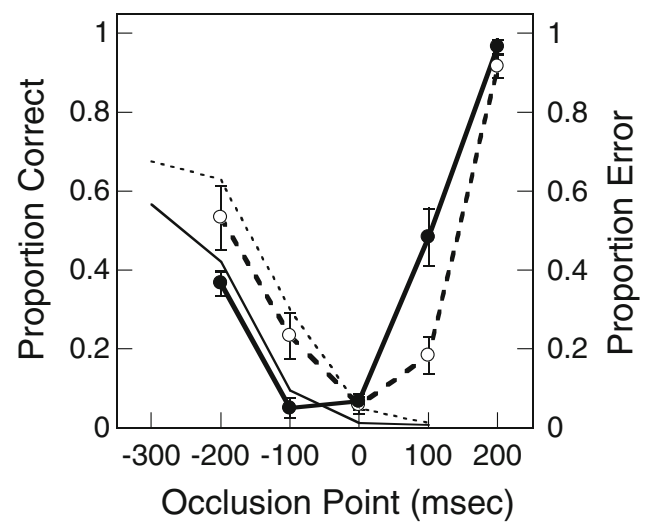


stimuli, the proportions correct of both groups increased more steeply, starting from below chance level, than for the no-sidestep stimuli. The players performed more accurately than the novices up to $0 \mathrm{~ms}$, after which the performances of both groups converged to near 1.0. For the doublesidestep stimuli, both the players and the novices showed peculiar patterns of performance. The proportions of correct responses of the two groups decreased to almost 0 as the occlusion point proceed up to $0 \mathrm{~ms}$, with the proportions of the novices being higher than those of the players. After the 0 -ms point, the proportions correct of both groups increased very steeply to almost 1.0 , with a higher rate of increase for the players than for the novices.

To verify these observations, we performed several ANOVAs on the individual proportions correct for the three types of stimuli. For the no- and one-sidestep stimuli, a 2 (group: player or novice) $\times 2$ (action: no or one sidestep) $\times 5$ (occlusion: $-300,-200,-100,0$, or $100 \mathrm{~ms}$ ) ANOVA showed significant main effects of action, $F(1,18)=75.90, \eta_{\mathrm{p}}^{2}=.808$, and occlusion, $F(4,72)=255.26, \eta_{\mathrm{p}}^{2}=.934$, both $p$ s $<.01$, while the main effect of group failed to reach significance, $F(1,18)=3.50, p=.08, \eta_{\mathrm{p}}^{2}=.163$. All interactions except for Group $\times$ Occlusion, $F(4,72)=1.31, \eta_{\mathrm{p}}^{2}=.068$, were significant: Group $\times$ Action, $F(1,18)=20.35, \eta_{\mathrm{p}}^{2}=.531$; Action $\times$ Occlusion, $F(4,72)=31.98, \eta_{\mathrm{p}}^{2}=.640$; and Group $\times$ Action $\times$ Occlusion, $F(4,72)=6.43, \eta_{\mathrm{p}}^{2}=.263$, all $p \mathrm{~s}<.01$. The lack of a significant group effect or Group $\times$ Occlusion interaction seems to reflect the opposing patterns of player-novice differences between the two types of actions, which cancelled out each other's effects. This inference was supported by the significant Group $\times$ Action $\times$ Occlusion interaction and the results of ANOVAs conducted separately for the two actions (see below). Post-hoc comparison showed that both the players and the novices yielded significantly lower proportions of correct responses for the one-sidestep action than for the nosidestep action, both $p \mathrm{~s}<.01$. More specifically, significantly lower proportions of correct responses for the one-sidestep action were observed among the player group at -300 and $200 \mathrm{~ms}$ and among the novice group at $-300,-200$, and $100 \mathrm{~ms}$, all $p \mathrm{~s}<.01$. A series of $t$ tests with Bonferroni corrections set at a significance level of .05 for each type of action showed that all proportions of correct responses of the players and the novices were significantly higher than chance level (.5) for the no-sidestep action, whereas for the onesidestep action, proportions of correct responses significantly higher than chance level were observed for both groups at 100,0 , and $100 \mathrm{~ms}$. For the novices, the proportions of correct responses at -300 and $-200 \mathrm{~ms}$ were significantly lower than chance level.

For the no-sidestep action, a two-factor ANOVA showed significant main effects of group, $F(1,18)=4.82, p<.05$, $\eta_{\mathrm{p}}^{2}=.211$, and occlusion, $F(4,72)=110.44, p<.01, \eta_{\mathrm{p}}^{2}=$. 860 , as well as a significant interaction between them, $F(4$, $72)=2.77, p<.05, \eta_{p}^{2}=.133$. Post-hoc comparisons showed that the proportions of correct responses were significantly higher for the novices than for the players only at the -200 -ms occlusion point, $p<.05$. For the novices, the proportion of correct responses significantly increased from -300 to both -200 and $-100 \mathrm{~ms}$, both $p \mathrm{~s}<.01$, after which the proportion of correct responses was nearly perfect and did not change significantly. The players demonstrated similar results, except that they showed no significant increase from -300 to $-200 \mathrm{~ms}$. For the one-sidestep action, we found significant main effects of group, $F(1,18)=15.07$, $\eta_{\mathrm{p}}^{2}=.456$, and occlusion, $F(4,72)=154.08, \eta_{\mathrm{p}}^{2}=.895$, as well as a significant interaction between them, $F(4,72)=4.24$, $\eta_{\mathrm{p}}^{2}=.191$, all $p \mathrm{~s}<.01$. Post-hoc comparisons showed significantly higher proportions for the players than for the novices at $-300,-200$, and $-100 \mathrm{~ms}$, all $p \mathrm{~s}<.05$. For the players, the proportion of correct responses increased significantly from 200 to $-100 \mathrm{~ms}, p<.01$, after which it leveled off near $100 \%$. The novices yielded similar results, except that they also showed a significant increase from -100 to $0 \mathrm{~ms}, p<.01$.

For the double-sidestep action, the main effect of group was not significant, $F(1,18)=0.01$, whereas the main effect of occlusion, $F(4,72)=111.68, \eta_{\mathrm{p}}^{2}=.861$, and the Group $\times$ Occlusion interaction, $F(4,72)=8.96, \eta_{\mathrm{p}}^{2}=.332$, were significant, both $p \mathrm{~s}<.01$. The nonsignificant effect of group was due to the opposing patterns of the player-novice difference before versus after the 0 -ms occlusion point, as indicated by the Group $\times$ Occlusion interaction. Post-hoc comparisons showed that the proportion of correct responses for the novices was higher than that for the players at $-100 \mathrm{~ms}$, $p<.01$, whereas the proportion of correct responses for the players was higher at $100 \mathrm{~ms}, p<.01$. For the players, the proportions of correct responses at -100 and $0 \mathrm{~ms}$ were significantly lower that those at any other occlusion points, all $p \mathrm{~s}<.01$. The proportion of correct responses significantly increased from 100 to $200 \mathrm{~ms}, p<.01$. For the novices, the proportion of correct responses decreased significantly from 200 to both -100 and $0 \mathrm{~ms}$, both $p \mathrm{~s}<.01$. Although the proportion of correct responses was not significantly different between 0 and $100 \mathrm{~ms}$, it was significantly higher at 200 than at $100 \mathrm{~ms}, p<.01$.

\section{Discussion}

The three types of running actions yielded markedly different patterns of player-novice differences in anticipation performance. This is in sharp contrast to the results of Experiment 1, in which the rugby players outperformed the novices in the RT task in all three types of actions, even 
though the actions used in the two experiments were basically identical. These results suggest that RT and temporalocclusion tasks reveal different aspects of anticipatory processing in a sports context, although they are usually regarded as methodologies probing the temporal characteristics of anticipation (Williams et al., 1999). Issues concerning the results obtained with the two paradigms will be discussed further in the General Discussion.

It is important to note that the player-novice difference was reversed between the no-sidestep and one-sidestep actions (top two panels of Fig. 4). For the one-sidestep actions, the players performed more accurately than the novices, while the players performed less accurately for the no-sidestep actions. The results for the one-sidestep action are consistent with those of Jackson et al. (2006) and Brault et al. (2012) for deceptive actions and are in good accord with the notion that expert players are less susceptible to deception than novices (Jackson et al., 2006). This is evident in the time course of anticipation performance shown in the top right panel of Fig. 4. Remember that the deceptive sidestep took place at around -400 to $-300 \mathrm{~ms}$ in the running action (Fig. 1). When the action was occluded at $-300 \mathrm{~ms}$, the novices performed significantly lower than chance level, which means that they were tricked by the deceptive motion and judged it to be a genuine step more often than as a deceptive one. The players also performed below chance level, but not significantly, and they performed significantly more accurately than the novices. This suggests that the players were relatively invulnerable to the deceptive motion, even when the action that they had seen contained only deceptive information. The novices continued to perform poorly at $-200 \mathrm{~ms}$, and they exceeded chance level at $-100 \mathrm{~ms}$ and afterward. The players, on the other hand, performed above chance level at $-200 \mathrm{~ms}$ and reached near $90 \%$ accuracy at $-100 \mathrm{~ms}$. Both groups performed almost perfectly at 0 and $100 \mathrm{~ms}$, when the occluded action contained a genuine step at 0 and $100 \mathrm{~ms}$. Altogether, the results clearly indicate that the players were superior to the novices in anticipating correctly on the basis of advance deceptive information (Jackson et al., 2006).

The seemingly inferior performance of the players for the no-step action is different from the findings in a majority of the sports perception research, including Jackson et al. (2006) and Brault et al. (2012), which have shown that expert players are superior to, or in some cases no different from, novices in anticipating future events (Williams et al., 1999). One possibility is that in this experiment the players expected to see deceptive actions more often than did the novices. From their long experience of the game, the players had learned that a running opponent carrying a ball is likely to use sidesteps. This knowledge would have led the players to have a high expectancy of deceptive actions when they saw opponents running toward them, biasing their judgments of directional change. This is consistent with a recent finding of Cañal-Bruland and Schmidt (2009) that goalkeepers are biased in judging penalty shots as fake. Such a bias lowers the proportions of correct responses for genuine actions, in this case the no-sidestep actions. The players would have mistaken a genuine step as a deceptive one and answered with the direction opposite to that indicated by the action, but their answer was wrong. On the other hand, the novices, without expert knowledge of rugby, would not have expected deceptive actions as much as the players; they would have judged the actions simply on the basis of the visual information contained in the action.

Another possibility is that the players' poor performance reflected their inferior perceptual ability to determine directional change from advance visual information in the nosidestep action. We think this explanation unlikely, however, for we find no reason why the players' experience and expertise knowledge of rugby would have a negative impact on their perception. Rather, we reasoned from our data that the perceptual abilities to anticipate the directional change of the no-sidestep action were not different between the players and the novices (Jackson et al., 2006). For both groups, the no-sidestep actions were easy to judge: Their proportions of correct responses were significantly higher than chance level at $-300 \mathrm{~ms}$ and increased to over $90 \%$ at $100 \mathrm{~ms}$. This suggests that the novices, as well as the players, would have already possessed the perceptual abilities necessary to correctly judge the directional change of the running action without deception. Because their perceptual abilities were equivalent, their differential expectancies of deception would have made their performance different.

It is likely that the players' expectancy also affected their judgments of deceptive actions. For example, their expectancy may have contributed to the players' relatively accurate performance for the one-sidestep action. Ample evidence in the attention literature has indicated that expectancy of objects or object attributes improves sensitivity and responses to the objects when they are presented (Kunde et al., 2007; Milliken \& Tipper, 1998).

A totally different pattern of results was obtained for the double-sidestep actions (bottom panel of Fig. 4). To account for these results, we hypothesize that the participants confused the double-sidestep actions with the one-sidestep actions until the 0 -ms occlusion point, after which they realized that the actions contained double sidesteps and judged them appropriately. Remember that for the doublesidestep actions, the occlusion point of $0 \mathrm{~ms}$ was set at the moment of the second sidestep touching on the ground, in a way equivalent to the occlusion timing of the one-sidestep actions (see the Method section of this experiment). This made the two actions indistinguishable up to $0 \mathrm{~ms}$. To illustrate, we plot the mean proportion errors for the one- 
sidestep actions (thin continuous and dotted lines) in the bottom panel of Fig. 4. For both the players and the novices, those proportions of errors for the one-sidestep actions closely follow the proportions of correct responses for the double-sidestep actions between -200 and $0 \mathrm{~ms}$, indicating that the double-sidestep actions were wrongly judged as one-sidestep actions during that period. For the doublesidestep actions, the proportions of correct responses improved at $100 \mathrm{~ms}$, to a greater degree for the players, indicating that they came to realize that the second step was a deception and started to judge appropriately. The players' proportion correct was near 1.0 at $200 \mathrm{~ms}$ because they knew that there was no more deception and were able to make a decision on the basis of the direction of the second sidestep; the correct answer was the direction opposite to that of the sidestep. The novices showed a similar pattern, but they were still in error at $100 \mathrm{~ms}$.

Interestingly, the participants mistook double-sidestep actions for one-sidestep actions, but not the reverse. The proportions of correct responses would have been at chance level for both types of actions if the wrong judgments were made equally for both of them. This suggests that the participants regarded the second step, whether it was genuine or fake, as genuine, without taking into consideration that it could be fake. This tendency may not be related to expert knowledge, because it was observed for the novices as well as the players, but it is likely to be due to the relative frequencies of the one-sidestep and double-sidestep actions: The former was presented twice as often as the latter.

Taken together, the results of this experiment imply that rugby players' anticipation of direction change of a running opponent involves two components. One is their perceptual ability to determine the direction from advance visual information contained in the running action. Their abilities may not be different from those of the novices when they are applied to nondeceptive actions, but the players' ability works better for deceptive actions. Another component is the expectancy of certain kinds of actions. The expectancy may originate from expert knowledge of deceptive actions in rugby and the relative frequencies of different types of actions presented in a given situation.

\section{Experiment 3}

In Experiment 3, the stimuli were point-light representations of the running actions used in Experiment 2 (see Fig. 5). If the results of Experiment 3 were to replicate the findings of Experiment 2, this would suggest that anticipatory cues utilized by experienced rugby players are kinematic - that is, an opponent's running "motion" per se, rather than contour or surface information from the opponent's body.

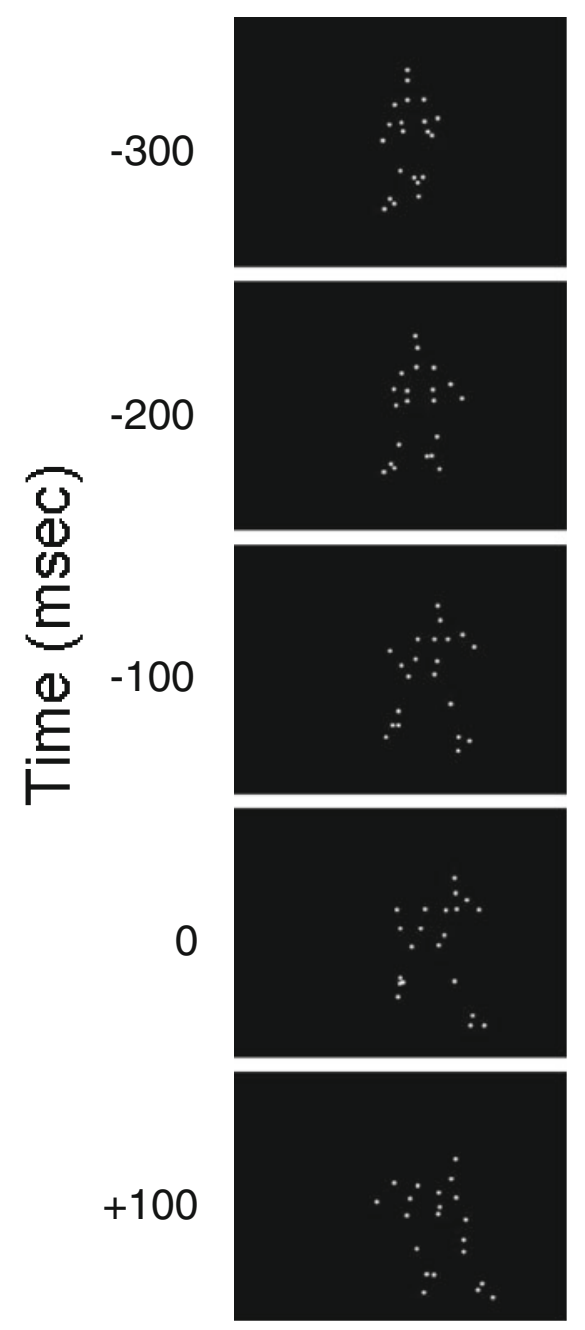

Fig. 5 Example of a point-light stimulus used in Experiment 3. The times on the left side indicate occlusion points. This stimulus is the representation of the one-sidestep actions shown in Fig. 1

\section{Method}

Ten collegiate rugby players and ten novices (mean ages $22.0 \pm 1.4$ years and $22.8 \pm 1.0$ years, respectively), all males, participated. They were different from those who had participated in the prior experiments of this study. The players had played rugby for a mean of $8.8 \pm 2.3$ years, with five of them having competed at the national level or been selected to a regional top team in Japan. The novices were university students who had not played rugby at a competitive level, but all of them had seen rugby played and possessed basic knowledge of the game. All of the participants had normal or corrected-to-normal acuity and color vision, and they gave informed written consent prior to the experiment.

The apparatus was the same as in Experiment 2. Pointlight stimuli were created from 30 video-recorded actions (12 no-sidestep, 12 one-sidestep, and 6 double-sidestep) 
used as stimuli in Experiments 1 and 2. Each stimulus consisted of a moving pattern of 21 disconnected points that corresponded to the coordinates of major landmarks of the body: the vertex and tragion of the head, the suprasternal, right, and left shoulders, and the elbows, middle knuckles, lower ribs, greater trochanters, knees, ankles, heels, and toes. Those coordinates were determined by digitizing the video-recorded action frame by frame with motion-analysis software (DKH, Frame Dias II) and were represented by small white circles against a background. The point-light stimuli were presented in the form of successive frames at $30 \mathrm{~Hz}$ on the screen, in the same manner as for the video stimuli of Experiments 1 and 2. An example point-light stimulus is shown in Fig. 5. Note that this point-light stimulus was created from the video stimulus of the one-sidestep action shown in Fig. 1: Each frame is a representation of the runner's body at the corresponding timing.

The task was identical to that of Experiment 2. The stimuli were presented until one of five occlusion points, set at timings identical to those of the corresponding video stimuli of Experiment 2 (see the Method section of that experiment). Before the experiment, the participants were told that the stimuli to be presented were point-light representations of rugby players' running actions, which might or might not include sidesteps. After the instruction, the participants were given demonstrations of point-light stimuli of three types of actions, each presented with no temporal occlusion. Each participant completed an experimental session of 6-12 practice trials and 150 main trials, in which the stimuli consisted of random presentations of the combinations of the 30 different actions and five occlusion points. The other procedures were identical to those of Experiment 2 .

\section{Results}

Figure 6 shows the mean proportions of correct responses of the rugby players and the novices as a function of occlusion points, separately for the three types of stimuli. For comparison, the results of Experiment 2 are also shown in each graph. Clearly, the results of this experiment replicated the main features of the results of Experiment 2. For the nosidestep actions, the novices yielded higher proportions than the players, whereas the players outperformed the novices for the one-sidestep actions. For those two actions, the proportions of correct responses increased from $-300 \mathrm{~ms}$ to $0 \mathrm{~ms}$, after which the proportions were near $100 \%$. For the double-sidestep actions, both the players and the novices
Fig. 6 Results of Experiment 3: Mean proportions of correct responses of ten rugby players and ten novices, presented separately for the three types of actions. The vertical bars indicate standard errors of the means. In each panel, the results of Experiment 2 are also shown as thin gray continuous (players) and dotted (novices) lines
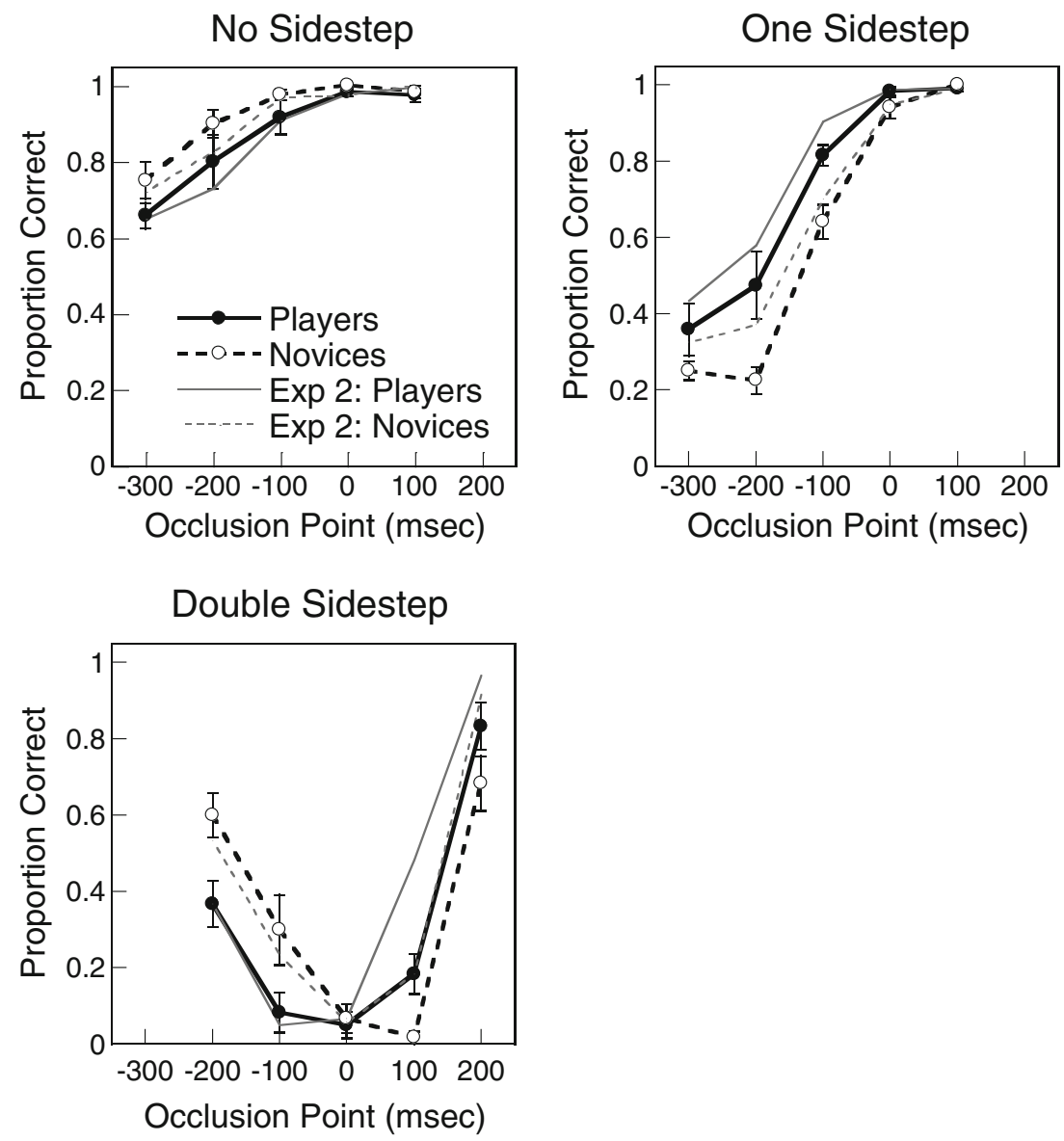
showed U patterns of proportions. The novices yielded relatively higher proportions than did the players up to $0 \mathrm{~ms}$, after which this difference was reversed.

To verify these observations, we performed ANOVAs and multiple comparisons, with the overall $\alpha$ level adjusted to .05 by Bonferroni corrections, on the individual proportions of correct responses separately for the three types of actions. For the no-sidestep actions, the main effect of occlusion was significant, $F(4,72)=36.92, p<.01, \eta_{\mathrm{p}}^{2}=.672$, but the main effect of group, $F(1,18)=2.40, \eta_{\mathrm{p}}^{2}=.118$, and the Group $\times$ Occlusion interaction, $F(4,72)=1.13, \eta_{\mathrm{p}}^{2}=.059$, were not. The lack of significance of the latter two effects appears to be due to the relatively large individual differences in responses to the no-sidestep actions, as indicated by error bars. Multiple comparisons on the occlusion effect showed that the proportions at $-300 \mathrm{~ms}$ and $-200 \mathrm{~ms}$ were both significantly lower than any of the proportions at $0 \mathrm{~ms}$ or later, all $p \mathrm{~s}<.05$, while no significant differences emerged between any of the proportions at 0,100 , and $200 \mathrm{~ms}$.

For the one-sidestep actions, the main effects of group, $F(1,18)=8.96, \eta_{\mathrm{p}}^{2}=.332$, and occlusion, $F(4,72)=$ $160.12, \eta_{\mathrm{p}}^{2}=.899$, were significant, both $p \mathrm{~s}<.01$, together with a significant interaction between them, $F(4,72)=3.94$, $p<.05, \eta_{\mathrm{p}}^{2}=.180$. The players' proportions were significantly higher than those of the novices at -200 and $-100 \mathrm{~ms}$, both $p \mathrm{~s}<.05$. For both groups, the proportions significantly increased from $-200 \mathrm{~ms}$ to both -100 and $0 \mathrm{~ms}$, all $p \mathrm{~s}<.01$, at which point the proportions leveled off near $100 \%$, so that there was no significant increase at $100 \mathrm{~ms}$.

For the double-sidestep actions, the main effect of occlusion, $F(4,72)=52.38, \eta_{\mathrm{p}}^{2}=.744$, and the Group $\times$ Occlusion interaction, $F(4,72)=5.48, \eta_{\mathrm{p}}^{2}=.234$, were significant, both $p \mathrm{~s}<.01$, but the main effect of group, $F(1,18)=0.80, \eta_{\mathrm{p}}^{2}=.043$, was not significant. The players' proportion of correct responses was significantly lower than that of the novices at $-200 \mathrm{~ms}, p<.05$, whereas the players' proportion of correct responses was significantly higher at $100 \mathrm{~ms}, p<.01$. For the players, the proportion of correct responses at $0 \mathrm{~ms}$ was significantly lower than the proportions of correct responses at either -200 or $200 \mathrm{~ms}$, both $p$ s $<.01$. The proportion of correct responses significantly increased from 100 to $200 \mathrm{~ms}, p<.01$. For the novices, the proportions of correct responses decreased significantly from -200 to both -100 and $0 \mathrm{~ms}$, both $p \mathrm{~s}<.05$. Although the proportion of correct responses was not significantly different between 0 and $100 \mathrm{~ms}$, a significant increase did emerge at $200 \mathrm{~ms}$ from $100 \mathrm{~ms}, p<.01$.

In order to compare the data from the video and the pointlight stimuli, we conducted separate three-way ANOVAs for each type of action, with Experiment (Exps. 2 and 3) and Group as between-participants factors, and Occlusion as a within-participants factor. We confined ourselves to the results relevant to the differences between the two types of stimuli (Experiment factor). For the no-sidestep actions, none of the main effect of experiment, $F(1,36)=0.88, \eta_{\mathrm{p}}^{2}=$. 024, or the interactions of Experiment $\times$ Group, $F(1,36)=$ $0.06, \eta_{\mathrm{p}}^{2}=.002$, Experiment $\times$ Occlusion, $F(4,144)=2.00$, $\eta_{\mathrm{p}}^{2}=.053$, or Experiment $\times$ Group $\times$ Occlusion, $F(4,144)=$ $0.45, \eta_{\mathrm{p}}^{2}=.001$, were significant. For the one-sidestep actions, we did find a significant main effect of experiment, $F(1,36)=$ $5.24, p<.05, \eta_{\mathrm{p}}^{2}=.127$, but no significant interactions of Experiment $\times$ Group, $F(1,36)=0.00, \eta_{\mathrm{p}}^{2}=.000$, Experiment $\times$ Occlusion, $F(4,144)=2.47, \eta_{p}^{2}=.064$, or Experiment $\times$ Group $\times$ Occlusion, $F(4,144)=0.15, \eta_{\mathrm{p}}^{2}=.004$. For the double-sidestep actions, a significant main effect of experiment emerged, $F(1,36)=9.84, p<.01, \eta_{\mathrm{p}}^{2}=.215$, as well as a significant Experiment $\times$ Occlusion interaction, $F(4,144)=$ $6.25, p<.01, \eta_{\mathrm{p}}^{2}=.148$, but no significant Experiment $\times$ Group, $F(1,36)=0.54, \eta_{\mathrm{p}}^{2}=.015$, or Experiment $\times$ Group $\times$ Occlusion, $F(4,144)=0.66, \eta_{\mathrm{p}}^{2}=.018$, interaction. Although the overall performance was better with the video than with the point-light stimuli for the one- and double-sidestep actions, the lack of a significant Experiment $\times$ Group and Experiment $\times$ Group $\times$ Occlusion interaction for either type of action supports that this experiment replicated the playernovice differences observed in Experiment 2.

\section{Discussion}

The remarkable similarity of the present data to the data of Experiment 2 indicates that the point-light stimuli of this experiment reproduced the critical features of the original video stimuli used in Experiments 1 and 2. As in previous studies comparing data from point-light stimuli with those from the original stimuli (Abernethy et al., 2001; Shim et al., 2006), the proportions of correct responses for the present point-light stimuli were equal to or lower than those for the video stimuli. However, the characteristic patterns of player-novice differences observed for the video stimuli were maintained for the point-light stimuli, suggesting that the anticipatory cues utilized by experienced rugby players are kinematic in nature.

\section{General discussion}

The present experiments demonstrated the spatiotemporal aspects of expert-novice differences in anticipating the direction changes of a running opponent in rugby. In Experiment 1, the experienced rugby players were faster than the novices in responding to direction changes in 
actions with and without sidesteps. Their eye movement data revealed that the players fixated mainly on the hips and legs of the running opponent, whereas the novices fixated mainly on the chest. In Experiment 2, using the temporal-occlusion paradigm, the players performed more accurately than did the novices for the one-sidestep actions before the direction change. In contrast, the players' performance was poorer than that of the novices for the nosidestep actions. Similar patterns of expert-novice differences were observed in Experiment 3 with point-light representations of the same running actions, suggesting that the anticipatory cues utilized by the players and the novices were kinematic in nature, and possibly the honest and deceptive signals of an opponent's running motion described by Brault and colleagues (Brault et al., 2010, 2012).

\section{Susceptibility to deception}

The present results support that rugby players are less susceptible than novices to sidesteps when they judge the direction changes of a running opponent (Brault et al., 2012; Jackson et al., 2006). This is clearly seen in the RT (Exp. 1) and temporalocclusion (Exps. 2 and 3) results for the one-sidestep actions: The rugby players responded significantly faster and made more accurate judgments than did the novices.

The present results also showed that the players' performance was impaired by the sidesteps. In Experiment 1, the players responded slightly but significantly slower to the one-sidestep action than to the no-sidestep actions. Their impairment was more evident in Experiment 2, where the players' overall performance was significantly less accurate for the one-sidestep actions than for the no-sidestep actions. The differences between those two actions were most marked at -300 and $-200 \mathrm{~ms}$, at which point the players' performance was around chance for the one-sidestep actions, while their performance was significantly above chance for the no-sidestep actions. The players' susceptibility to the sidesteps was also noted in their performance for the doublesidestep actions, which showed that the players were unable to distinguish the double-sidestep from the one-sidestep actions before the second sidestep was completed. These findings were replicated in Experiment 3 with the point-light displays. We thus concluded that rugby players are susceptible to deceptive actions when they judge direction changes of a running opponent, but less so than novices are.

Our conclusion is in line with recent studies on anticipation from deceptive action (Brault et al., 2010, 2012; Dicks et al., 2010a; Rowe et al., 2009) and is also consistent with a general expectancy for deceptive actions to be employed by experienced players - that is, deceptive actions are intended to mislead expert opponents, not novices. Players exercise and refine their deceptive actions in order to make them effective on the field. The opponents attempt to counteract this deception by developing their cognitive skills. Therefore, deceptive actions should be effective, but not perfectly so, on expert opponents. Whether this contention can be generalized to other sports is a matter for further investigation, since the purpose and use of deceptive actions vary with sports and with the levels of expertise of players (Cañal-Bruland \& Schmidt, 2009).

We also found that the effects of sidesteps may extend to the players' judgments of actions without sidesteps. In Experiments 2 and 3, the players were less accurate than the novices in anticipating the no-sidestep actions. This is different from the results of Jackson et al. (2006) and Brault et al. (2012), which showed equivalent performance among players and novices. Jackson et al. reasoned that judging the direction change of running is based on general perceptual skills possessed equally by players and novices. Their reasoning is partially supported by the present results, in which the players and the novices performed significantly above chance as early as $300 \mathrm{~ms}$ before the step to change direction. However, it is unlikely that the present findings reflect only the perceptual skills of discriminating running directions; if that were the case, the results for the no-sidestep actions would mean that the novices would be perceptually superior to the players. Rather, we propose that the player's seemingly poor performance is a byproduct of a judgment bias resulting from their expectation of deceptive actions (Cañal-Bruland \& Schmidt, 2009; see also the Discussion of Exp. 2). It is not clear whether such an expectation affected the players' judgments in Jackson et al.'s (2006) and Brault et al.'s (2012) studies, which did not show lower performance for players than for novices. One possible reason for the different findings obtained in the present study and in those two studies is the inclusion of the double-sidestep actions in the present study, which might have made the players' decision-making complicated and made them cautious about not being fooled by deceptive actions, whether the actions were with one or a double sidestep. This could have enhanced their judgment bias toward the sidestep actions, resulting in their relatively low performance for the no-sidestep actions. Such an enhancement of players' bias would not have occurred in Jackson et al.'s (2006) experiment using filmed sequences of running actions or in Brault et al.'s (2012) experiments conducted in an immersive virtual reality environment, so that the players' expectation of sidesteps in those experiments, if any, might not have been high enough to alter their performance for the nosidestep actions. This reasoning is speculative at best, and needs to be examined in further studies.

Discrepancies between the RT and temporal-occlusion paradigms

The present study revealed inconsistencies between the data of the RT (Exp. 1) and the temporal-occlusion (Exp. 2) 
paradigms, both of which were used to examine temporal characteristics of anticipation. While the RT data showed that the players were faster than novices, irrespective of whether or not the running actions involved sidesteps, the occlusion data showed the players' superior anticipation - that is, accurate performance prior to the genuine step for direction changeonly for the one-sidestep action. The players were less accurate than the novices for the no-sidestep actions. This discrepancy may be attributable to the response modes of the two paradigms, depending on whether a greater emphasis was made on the speed (RT paradigm) or accuracy (temporal-occlusion paradigm) of the participant's responses. It has been shown that expert players' advantages over novices become more marked as the response mode of the task simulates more closely the action required on the field (Farrow \& Abernethy, 2003; Mori et al., 2002; Williams et al., 1994), and the present findings are consistent with this contention. Farrow et al. (2005) showed that tasks involving different response modes - for instance, verbal responses with little time constraint and speeded actions on the field - yielded essentially the same results in temporalocclusion paradigms, although those tasks were different in other respects.

\section{Future directions}

The present findings add to our current understanding of anticipation and deception in sports expertise. To further advance this line of research, we may need to consider modifications of the methodologies used here. For example, the stimuli used in the present experiments were constructed from the actions of three model players. Although this followed the stimulus construction that has been used in a large number of sports anticipation studies, including Jackson et al. (2006), it could lead to questions about the generality of stimuli made from such a small number of model players and possible variations in the data due to their different actions. We reanalyzed all of the data of the present experiments with respect to the stimuli constructed from different model players and found that the stimuli made from one of the model players yielded significantly faster RTs (Exp. 1) and higher accuracies (Exps. 2 and 3) than did those from the other players, which yielded generally similar results to each other. No significant difference due to the model player was obtained in the eye movement data of Experiment 1. It thus seems that the actions of one player were easier to judge than those of the other two. We should note that the differential effects due to the model players did not interact with the effects of the participant group (player or novice) or with the interactions involving the Group factor in any of the three experiments. Therefore, the overall conclusions of the present study do not change if we consider the differences due to the model players. Nonetheless, future research needs to carefully consider the stimuli constructed from different model players.
The stimulus presentation and the participant's response mode in the present experiments may also need refinement. In the present experiments, all of the stimuli were presented on the screen, and thus lacked the three-dimensional perspectives that would be present when the actions were seen on the field. The responses were made by buttonpresses while the participant was seated in a chair, unlike the tackling actions done by rugby players. In this regard, there has been an increasing emphasis on an in-situ paradigm, in which players' anticipatory performance is examined in terms of their requisite actions in response to opponents' movements or to ball flight in very naturalistic environments of their sports (Dicks et al., 2010a; Dicks, Button, \& Davids, 2010b; Pinder, Davids, Renshaw, \& Araújo, 2011). Obviously the in-situ paradigm is more realistic than a so-called video simulation paradigm, such as the one used in the present study, and are expected to yield results that better represent the player's anticipation on the field. Similar advantages over the video simulation paradigm have also been reported in studies conducted in immersive, interactive virtual reality environments (Brault et al., 2012; Craig, Bastin, \& Montagne, 2011; Dessing \& Craig, 2010). While some common findings have been obtained with the in-situ, virtual reality, and video simulation paradigms, important differences in players' anticipatory performance between these paradigms have also been reported (Craig et al., 2011; Dicks et al., 2010a, b; Mann, Abernethy, \& Farrow, 2010; Mann, Williams, Ward, \& Janelle, 2007; Pinder et al., 2011). For example, Dicks et al. (2010b) found that experienced goalkeepers fixated the ball and the kicker's body for equal amounts of time when they anticipated the directions of penalty kicks in the in-situ paradigm, while they fixated the kicker's body longer than the ball in the video simulation paradigm. Such differences are likely also to affect the anticipatory performance of rugby players, and the generality of the present findings needs to be examined in future research with the in-situ and/or the virtual reality paradigm.

Author Note The experiments reported in this article were conducted by the second author as part of a master's thesis submitted to Kyushu University. Some of the data have been previously reported, in a somewhat different form, at the 25th Annual Meeting of the International Society for Psychophysics, Galway, Ireland, October 2009. The research was supported by Grants-in-Aid for Scientific Research 17530528 and 21330169 to S.M. We thank Denis O'Hora, Takayuki Higuchi, Makiko Sadakata, Minoru Karasawa, and Nobuyuki Hirose for valuable suggestions. We also thank Cathy M. Craig and Simon Grondin for their helpful comments on early versions of this article.

\section{References}

Abernethy, B. (1990). Anticipation in squash: Differences in advance cue utilization between expert and novice players. Journal of Sport Science, 8, 17-34. 
Abernethy, B., Gill, D., Parks, S., \& Packer, S. (2001). Expertise and the perception of kinematic and situational probability information. Perception, 30, 233-252.

Abernethy, B., \& Russell, D. G. (1987a). Expert-novice differences in an applied selective attention task. Journal of Sport Psychology, 9, 326-345.

Abernethy, B., \& Russell, D. G. (1987b). The relationship between expertise and visual search strategy. Human Movement Science, 6, 283-319.

Abernethy, B., \& Zawi, K. (2007). Pickup of essential kinematics underpins expert perception of movement patterns. Journal of Motor Behavior, 39, 353-367.

Abernethy, B., Zawi, K., \& Jackson, R. C. (2008). Expertise and attunement to kinematic constraints. Perception, 37, 931-948.

Besson, M., \& Faïta, F. (1995). An event-related potential (ERP) study of musical expectancy: Comparison of musicians with nonmusicians. Journal of Experimental Psychology. Human Perception and Performance, 21, 1278-1296.

Blake, R., \& Shiffrar, M. (2007). Perception of human motion. Annual Review of Psychology, 58, 47-73. doi:10.1146/ annurev.psych.57.102904.190152

Bond, C. F., Jr., \& DePaulo, B. M. (2006). Accuracy of deception judgments. Personality and Social Psychology Review, 10, 213-234.

Brault, S., Bideau, B., Craig, C., \& Kulpa, R. (2010). Balancing deceit and disguise: How to successfully fool the defender in a 1 vs. 1 situation in rugby accuracy of deception judgments. Human Movement Science, 29, 412-425.

Brault, S., Bideau, B., Kulpa, R., \& Craig, C. (2012). Detection deception in movement: The case of the side-step in rugby. PLoS One, 7, 1-13.

Cañal-Bruland, R., \& Schmidt, M. (2009). Response bias in judging deceptive movements. Acta Psychologica, 130, 235-240.

Cauraugh, J. H., \& Janelle, C. M. (2002). Visual search and cue utilization in racket sports. In K. Davids, G. J. P. Savelsbergh, S. J. Bennett, \& J. van der Kamp (Eds.), Interceptive actions in sport (pp. 64-89). London, UK: Routledge.

Coull, J. T., \& Nobre, A. C. (1998). Where and when to pay attention: The neural systems for directing attention to spatial locations and to time intervals as revealed by both PET and fMRI. Journal of Neuroscience, 18, 7426-7435.

Craig, C. M., Bastin, J., \& Montagne, G. (2011). How information guides movement: Intercepting curved free kicks in soccer. Human Movement Science, 30, 931-941. doi:10.1016/ j.humov.2010.08.007

Dessing, J. C., \& Craig, C. M. (2010). Bending it like Beckham: How to visually fool the goalkeeper. PLoS One, 5(10), e13161, 1-8. doi:10.1371/journal.pone.0013161

Diaz, G. J., Fajen, B. R., \& Phillips, F. (2012). Anticipation from biological motion: The goalkeeper problem. Journal of Experimental Psychology. Human Perception and Performance, 38, 848-864. doi:10.1037/a0026962

Dicks, M., Button, C., \& Davids, K. (2010a). Availability of advance visual information constrains association-football goalkeeping performance during penalty kicks. Perception, 39, 1111-1124.

Dicks, M., Button, C., \& Davids, K. (2010b). Examination of gaze behaviors under in situ and video simulation task constraints reveals differences in information pickup for perception and action. Attention, Perception, \& Psychophysics, 72, 706-720. doi:10.3758/APP.72.3.706

Farrow, D., \& Abernethy, B. (2003). Do expertise and the degree of perception-action coupling affect natural anticipatory performance? Perception, 32, 1127-1139.

Farrow, D., Abernethy, B., \& Jackson, R. C. (2005). Probing expert anticipation with the temporal occlusion paradigm: Experimental investigations of some methodological issues. Motor Control, 9, $332-351$
Hagemann, N., Schorer, J., Cañal-Bruland, R., Lotz, S., \& Strauss, B. (2010). Visual perception in fencing: Do the eye movements of fencers represent their information pickup? Attention, Perception, \& Psychophysics, 72, 2204-2214. doi:10.3758/BF03196695

Helsen, W. F., \& Starkes, J. L. (1999). A multidimensional approach to skilled perception and performance in sport. Applied Cognitive Psychology, 13, 1-27. doi:10.1002/(SICI)10990720(199902)13:1<1::AID-ACP540>3.0.CO;2-T.

Huys, R., Smeeton, N. J., Hodges, N. J., Beek, P. J., \& Williams, A. M. (2008). On the dynamic information underlying visual anticipation skill. Attention, Perception, \& Psychophysics, 70, 12171234. doi:10.3758/PP.70.6.1217

Jackson, R. C., Warren, S., \& Abernethy, B. (2006). Anticipation skill and susceptibility to deceptive movement. Acta Psychologica, 123, 355-371.

Janata, P., \& Reisberg, D. (1988). Response-time measures as a means of exploring tonal hierarchies. Music Perception, 6, 163-174.

Johansson, G. (1973). Visual perception of biological motion and a model for its analysis. Perception \& Psychophysics, 14, 201-211. doi:10.3758/BF03212378

Jones, C. M., \& Miles, T. R. (1978). Use of advance cues in predicting the flight of a lawn tennis ball. Journal of Human Movement Studies, 4, 231-235.

Krumhansl, C. L., \& Shepard, R. N. (1979). Quantification of the hierarchy of tonal functions within a diatonic context. Journal of Experimental Psychology. Human Perception and Performance, 5, 579-594. doi:10.1037/0096-1523.5.4.579

Kunde, W., Elsner, K., \& Kiesel, A. (2007). No anticipation-no action: The role of anticipation in action and perception. Cognitive Process, 8, 71-78.

Loui, P., \& Wessel, D. (2007). Harmonic expectation and affect in Western music: Effects of attention and training. Perception \& Psychophysics, 69, 1084-1092.

Mann, D. L., Abernethy, B., \& Farrow, D. (2010). Action specificity increases anticipatory performance and the expert advantage in natural interceptive tasks. Acta Psychologica, 135, 17-23. doi:10.1016/j.actpsy.2010.04.006

Mann, D. L., Williams, M. A., Ward, P., \& Janelle, C. M. (2007). Perceptual-cognitive expertise in sport: A meta-analysis. Journal of Sport \& Exercise Psychology, 29, 457-478.

Milliken, B., \& Tipper, S. P. (1998). Attention and inhibition. In H. Pashler (Ed.), Attention (pp. 239-243). Hove, UK: Psychology Press.

Mori, S., Ohtani, Y., \& Imanaka, K. (2002). Reaction times and anticipatory skills of karate athletes. Human Movement Science, $21,213-230$.

Nobre, A. C., Correa, A., \& Coull, J. T. (2007). The hazard of time. Current Opinion in Neurobiology, 17, 1-6.

Osterhout, L., \& Holcomb, P. J. (1992). Event-related brain potentials elicited by syntactic anomaly. Journal of Memory and Language, 31, 785-806.

Paull, G., \& Glencross, D. (1997). Expert perception and decision making in baseball. International Journal of Sport Psychology, $28,35-56$

Pinder, R. A., Davids, K., Renshaw, I., \& Araújo, D. (2011). Manipulating informational constraints shapes movement reorganization in interceptive actions. Attention, Perception, \& Psychophysics, 73, 1242-1254. doi:10.3758/s13414-011-0102-1

Posner, M. I. (1980). Orienting of attention. Quarterly Journal of Experimental Psychology, 32, 3-25. doi:10.1080/00335558008248231

Rowe, R., Horswill, M. S., Kronvall-Parkinson, M., Poulter, D. R., \& McKenna, F. P. (2009). The effect of disguise on novice and expert tennis players' anticipation ability. Journal of Applied Sport Psychology, 21, 178-185.

Runeson, S., \& Frykholm, G. (1983). Kinematic specification of dynamics as an informational bias for person-and-action perception: 
Expectation, gender recognition, and deceptive intention. Journal of Experimental Psychology. General, 112, 585-615.

Savelsbergh, G. J. P., Williams, A. M., van der Kamp, J., \& Ward, P. (2002). Visual search, anticipation and expertise in soccer goalkeepers. Journal of Sports Sciences, 20, 279-287.

Sebanz, N., \& Shiffrar, M. (2009). Detecting deception in a bluffing body: The role of expertise. Psychonomic Bulletin \& Review, 16 , 170-175. doi:10.3758/PBR.16.1.170

Seya, Y., \& Mori, S. (2007). Tradeoff between response speed and pursuit accuracy. Motor Control, 11, 109-118.

Seya, Y., \& Mori, S. (2012). Spatial attention and reaction times during smooth pursuit eye movement. Attention, Perception, \& Psychophysics, 74, 493-509. doi:10.3758/s13414-011-0247-y

Shim, J., Carlton, L. G., \& Kwon, Y.-H. (2006). Perception of kinematic characteristics of tennis strokes for anticipating stroke type and direction. Research Quarterly for Exercise and Sport, 77, 326-339.

Vignais, N., Kulpa, R., Craig, C., Brault, S., Multon, F., \& Bideau, B. (2010). Influence of the graphical levels of detail of a virtual thrower on the perception of the movement. Presence, 19, 243252.

Ward, P., Williams, A. M., \& Bennett, S. J. (2002). Visual search and biological motion perception in tennis. Research Quarterly for Exercise and Sport, 73, 107-112.

Williams, A. M., \& Burwitz, K. (1993). Advance cue utilization in soccer. In T. Reilly, J. Clarys, \& A. Stribbe (Eds.), Science and football, Vol. II (pp. 239-243). London, UK: E \& FN Spon.

Williams, A. M., \& Davids, K. (1998). Visual search strategy, selective attention and expertise in soccer. Research Quarterly for Exercise and Sport, 69, 111-129.

Williams, A. M., Davids, K., Burwitz, K., \& Williams, J. G. (1994). Visual search strategies of experienced and inexperienced soccer players. Research Quarterly for Exercise and Sport, 65, 127-135.

Williams, A. M., Davids, K., \& Williams, J. G. P. (1999). Visual perception and action in sport. London, UK: E \& FN Spon.

Wright, R. D., \& Ward, L. M. (2008). Orienting of attention. New York, NY: Oxford University Press. 